\title{
Traumatic brain injury and sleep disturbances in combat-exposed service members and veterans: Where to go next?
}

\author{
Ashlee B. McKeon ${ }^{\mathrm{a}, *}$, Ryan P.J. Stocker ${ }^{\mathrm{b}}$ and Anne Germain ${ }^{\mathrm{a}}$ \\ ${ }^{a}$ University of Pittsburgh School of Medicine, Pittsburgh, PA, USA \\ ${ }^{\mathrm{b}}$ University of Pittsburgh Medical Center, Pittsburgh, PA, USA
}

\begin{abstract}
.
OBJECTIVE: To synthesize the current evidence on sleep disturbances in military service members (SMs) and veterans with traumatic brain injury (TBI).

METHODS: An electronic literature search first identified abstracts published from 2008-2018 inclusively referencing sleep, TBI, and military personnel from Operation Enduring Freedom, Operation Iraqi Freedom, Operation New Dawn, and Persian Gulf veterans. Selection criteria eliminated studies on non-combat TBI, open or penetrating injuries, and articles where the relationship between sleep and TBI was not directly examined. Articles on all military branches and components, those currently serving and veterans-ranging from medical chart reviews to clinical trials, were included. Forty-one articles were selected for full text-review.

RESULTS: Twenty-four papers estimated the prevalence of sleep disturbances in TBI. Eight studies demonstrated the contribution of common co-occurring conditions, most notably posttraumatic stress disorder, to the relationship between disrupted sleep and TBI. Ten studies differentiated sleep profiles between military SMs and veterans with and without acute TBI and detected significant differences in sleep disturbances across the course of injury. Longitudinal studies were scarce but helped to establish the temporal relationship between sleep disturbances and TBI and isolate sleep-related mechanisms influencing TBI prognosis. Only three studies reported on interventions for improving sleep quality and TBI symptoms. Systematic research testing assessments and interventions that target sleep disturbances for improving sleep, TBI symptoms, and long-term functional outcomes were identified as critical knowledge gaps.

CONCLUSION: Findings unequivocally establish that sleep disturbances are highly prevalent in SMs and veterans with TBI. However, studies testing the effectiveness of treatments for improving sleep in military groups with TBI have been limited and their results inconsistent. This review highlights a critical opportunity for advancing military medicine through future research aimed at identifying and testing sleep-focused treatments in SMs and veterans with combat-related TBI.
\end{abstract}

Keywords: Sleep disturbance, traumatic brain injury, military, service members, veterans, Operation Iraqi Freedom, Operation Enduring Freedom, Operation New Dawn, Persian Gulf veterans

\footnotetext{
*Address for correspondence: CPT Ashlee B. McKeon, Military Sleep Tactics and Resilience Research Team, University of Pittsburgh School of Medicine, 3811 O'Hara Street, Pittsburgh, PA 15213, USA. Tel.: +1 716400 1058; E-mail: ashlee.b.mckeon. mil@mail.mil.
}

\section{Background}

Traumatic brain injury (TBI) has been broadly defined by both the World Health Organization (WHO) and the United States Centers for Disease Control and Prevention (CDC) as the occurrence of a head injury from an external physical force after 
which at least one of the following symptoms manifests as a direct result: alteration in level of consciousness; retrograde or posttraumatic amnesia (PTA); other alterations in neurologic or neuropsychological function; cranial fracture; intracranial lesions; or death (Thurman, Alverson, Dunn, Guerrero, \& Sniezek, 1999). The majority of the literature on TBI has been conducted in either the communitydwelling adults or inpatient populations. However, military Service Members (SMs) and veterans represent a group at higher risk for TBI than observed in the general population (James, Strom, \& Leskela, 2014).

Between 2000-2015 alone, the incident rate of TBI sustained by members of the US Armed Forces reached 375,230 cases (Defense and Veterans Brain Injury Center, 2017). These numbers are believed to be underestimated due to prevalence estimates being based solely on individuals who report a TBI and receive treatment. Although approximately 82 percent of all TBIs experienced by SMs are considered 'mild' and have an average 10-day period before full return to duty, residual symptoms often manifest and become chronic (e.g., sleep disturbances; $\mathrm{Si}$ et al 2018). A report by the Veterans Health Administration (VHA) estimated that the health care costs for SMs with TBI are approximately four to six times as much during the first year of treatment as SMs without a TBI-over 60 percent of the total funds received (Congressional Budget Office, 2012). From 2004 to 2009 alone, combined costs related to TBI and PTSD health care for veterans in the first year of treatment was estimated to be 2.2 billion dollars (Congressional Budget Office, 2012). Thus, TBI is considered a hallmark operational stress injury associated with the Global War on Terror (Armed Forces Health Surveillance Center, 2013). Although the etiology of TBIs experienced by SMs extends beyond injuries resulting directly from combat (e.g., during training or in garrison), this paper focuses solely on TBIs resulting from combat exposure (e.g., blast and blunt force head trauma) due to blast and blunt-force injuries being the leading cause of TBI in warzones and the impact of sustaining a TBI during deployment operations on long-term individual outcomes and force readiness.

\subsection{Sleep disturbances in military populations}

Deployed active duty SMs are expected to perform optimally in high-risk operational environments where maintaining a normative sleep schedule is often not possible, such as night missions or forced extended wakefulness during multi-day missions. Further, chronic insufficient sleep has been shown to compromise safety, which suggests sleep restricted combat-exposed soldiers may be at an increased risk for sustaining mission-related injuries that compromise individual and unit-level readiness (e.g., TBI).

Although sleep loss is inherent to operational missions, chronic sleep loss is also prevalent among non-deployed SMs. Military personnel report sleep disturbances (e.g., insufficient or non-restorative sleep, insomnia, nightmares) before, during, and after deployment at alarming rates (Breen, Blankley, \& Fine, 2017; Caldwell, Knapik, \& Lieberman, 2017; de Dassel, Wittmann, Protic, Hollmer, \& Gorzka, 2017; Mysliwiec et al, 2013; Troxel et al, 2015). Numbers have continuously grown over the years and have well surpassed prevalence and incidence rates of the general population. Further, upwards of $75 \%$ of deployed SMs rate the quality of their sleep as significantly worse while in theater when compared to before deployment (Peterson, Goodie, Satterfield, \& Brim, 2008), many of whom meet for clinically significant insomnia. It is estimated that over $87 \%$ of SMs report at least one sleep disturbance, with the most commonly reported being insomnia, sleep fragmentation, obstructive sleep apnea, and hypersomnia (Bramoweth \& Germain, 2013; Mysliwiec, Gill, et al., 2013; Schiehser et al., 2017). Approximately $40 \%$ of soldiers report more than one sleep disturbance (Gill et al., 2014; Mysliwiec, Gill, et al., 2013), making the selection of targeted treatments exponentially more complex. Sleep disturbances are a critical issue facing the US military due to their impact on maintaining force readiness, health, wellbeing (Caldwell et al., 2017). Further, the economic burden of providing both acute and long-term behavioral health services related sleep disturbances has become a major concern. It is important to note that studies examining prevalence of sleep disturbances in military samples often ignore, or at the very least downplay, the contribution of pre-military service or pre-deployment sleep factors. Therefore, greater representation of baseline sleep parameters is needed in future research in order to fully examine the trajectory of sleep disturbances in military groups.

\subsection{Sleep disturbances in combat-related TBI}

Sleep disturbances are one of the most prevalent sequelae of TBI (AFHSC, 2013). In a sample of 114 post deployment military SMs and veterans 
who had sustained blast-related mTBI, 77\% endorsed experiencing sleep disturbances (Farrell-Carnahan, Franke, Graham, \& McNamee, 2013). Collen and colleagues reported that $97 \%$ of soldiers with TBI reported at least one sleep disturbance (Collen, Orr, Lettieri, Carter, \& Holley, 2012). These selfreported sleep disturbances were corroborated by polysomnographic sleep/wake studies and included hypersomnia (85\%), insomnia (55\%), sleep fragmentation $(54 \%)$, and obstructive sleep apnea syndrome (OSAS; 35\%). Sleep disturbances were more severe among SMs with comorbid TBI and posttraumatic stress symptoms (PTSS) or anxiety. There is preliminary evidence that blast- vs. blunt force-related TBI may be accompanied by distinct sleep disturbance profiles. Specifically, Kempft and colleagues reported that blast injuries were associated with insomnia and elevated anxiety symptoms, whereas blunt force injuries were more commonly associated with OSAS. These sleep disturbances are chronic: Over $60 \%$ of those with TBI continued to endorse clinically significant sleep disturbances three years post-injury (Kempf, Werth, Kaiser, Bassetti, \& Baumann, 2010).

Sleep disturbances can also impede the TBI rehabilitation process (Bell et al., 2018; Sandsmark, Elliott, \& Lim, 2017) and are risk factors for the development of anxiety and mood disorders following stress exposure (Babson \& Feldner, 2010; Bryant, Creamer, O'Donnell, Silove, \& McFarlane, 2010; Gehrman et al., 2013). Realizing the impact TBI has on functional outcomes and quality of life, as well as the role of disturbed sleep as a risk factor for the development and maintenance of death, disease, and disability, the comorbidity between TBI and chronic sleep disturbance may increase the risk for poor health outcomes among SMs and veterans relative to the general population (Barr et al., 2015).

The purpose of this review is to synthesize the literature focusing on sleep disturbances and TBI as it pertains specifically to military SMs and veterans. This review uniquely contributes to the existing science due to the overall scarcity of prior summaries of sleep disturbances and TBI, as well as existing reviews focusing primarily on the general population (see Barshikar \& Bell, 2017; Bogdanov, Naismith, \& Lah, 2017; Orff, Ayalon, \& Drummond, 2009; Ponsford et al., 2012 for reviews), on sleep disturbances occurring post-TBI (Bogdanov et al., 2017; Ponsford et al., 2012), or that isolate only one sleep disturbance (i.e. insomnia; Sandsmark, Elliott, \& Lim, 2017). First, we discuss the nature and prevalence of sleep disturbances based on varying TBI assessment meth- ods. Specific sleep profiles that may guide diagnostic and treatment efforts targeting disruptive sleep in TBI relative to other diagnostic groups are then suggested. Second, a summary of findings regarding the role of sleep in the trajectory of TBI is presented. Finally, critical knowledge gaps that, if filled, have a high potential for enhancing sleep health and functional outcomes in SMs and veterans with TBI are discussed.

\section{Methods}

An electronic scoping review of the literature on sleep and TBI in military SMs and veterans was conducted using the PubMed, PsychInfo, and Google Scholar databases. Sleep, TBI, and military were identified as three search term categories and the following terms were used in combination: "sleep", "sleep disturbance", "TBI", "traumatic brain injury", "closed head injury", "military", "servicemembers", "veterans", "Operation Enduring Freedom" (OEF), "Operation Iraqi Freedom" (OIF), "Operation New Dawn" (OND) and "Persian Gulf War" to generate the initial search. The search was limited to peerreviewed, full-text publications printed in English from January 2008 through January 2018 inclusively. The beginning year for the selection range was determined based on the exponential growth of studies focused on post-9/11 military groups extending from that time and previous reports summarizing the literature prior to 2008. A total of 63 published manuscript were found. Abstracts were reviewed by the first and second authors, and only those containing at least one search term from all three categories (i.e. sleep, TBI, military) were considered. Selection criteria then eliminated studies on non-combat TBI, open or penetrating injuries, or articles where the relationship between sleep and TBI was not directly examined. Articles on all military branches and components, those currently serving and veterans-ranging from reviews to clinical trials, were included. A total of 41 articles went through full-text review. These studies are summarized in detail in Table 1.

\section{Summary of the literature}

\subsection{Time course of sleep disturbances \& TBI}

Two of the 41 papers selected explicitly sought to evaluate the time course between sleep disturbance 


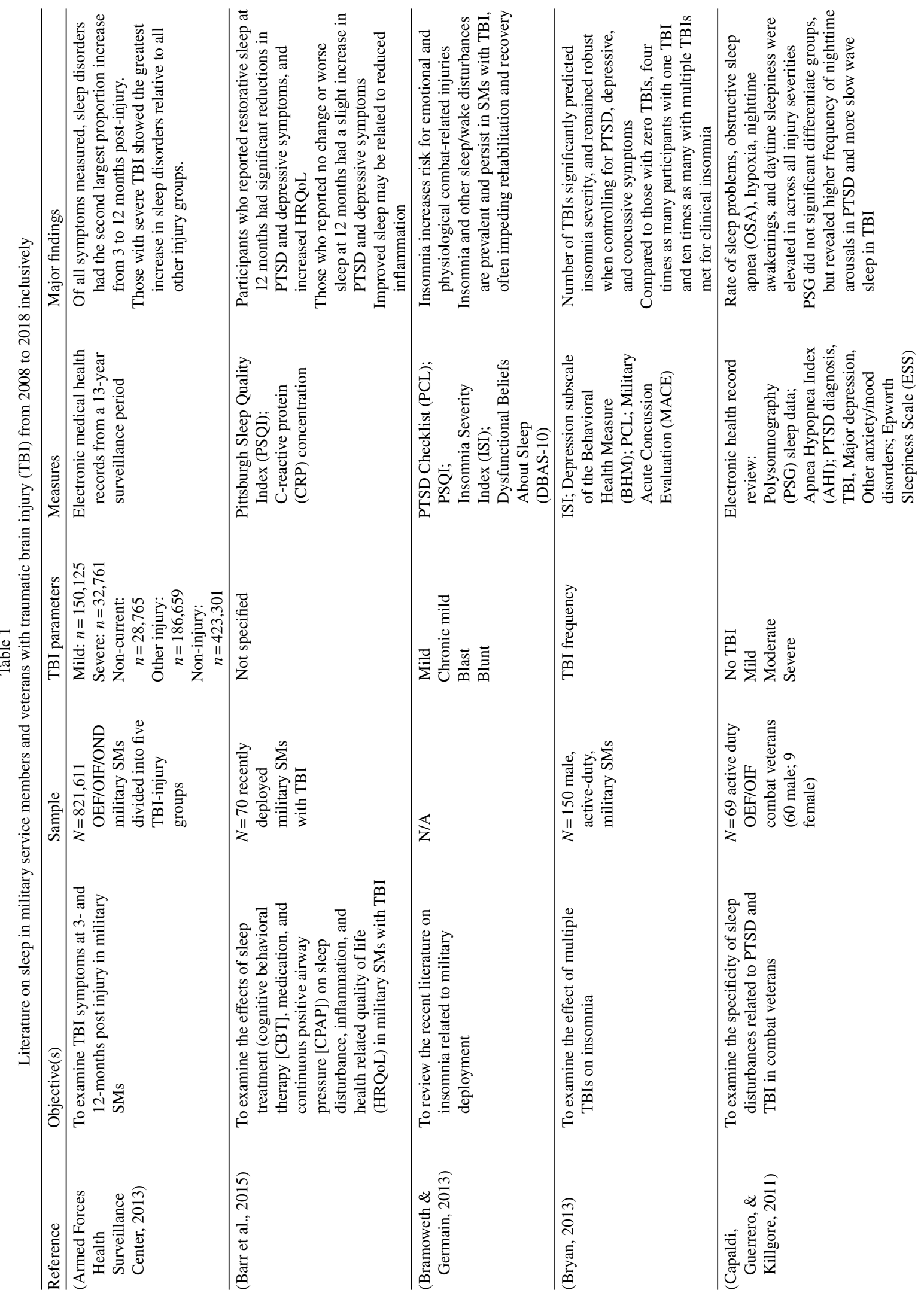



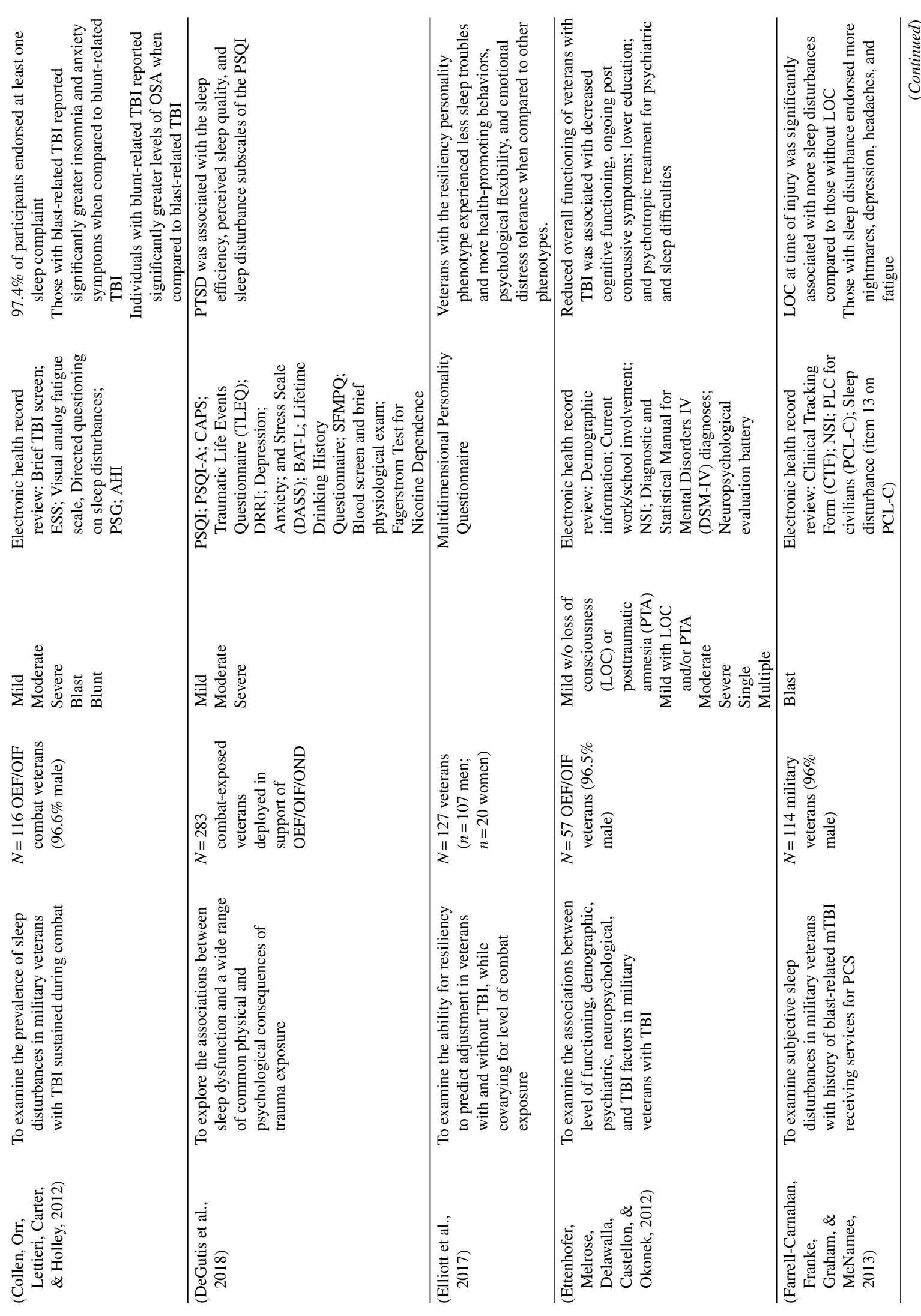


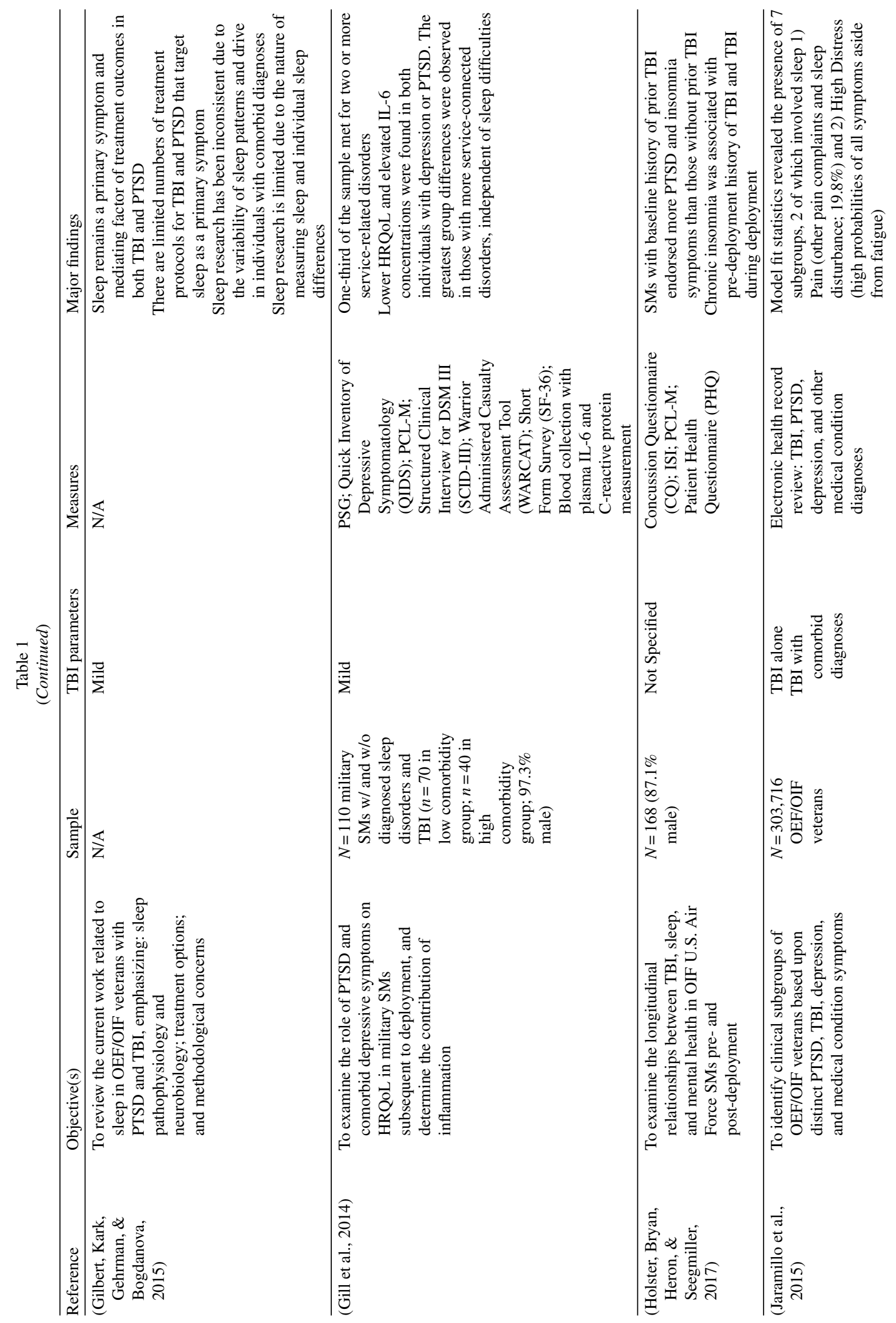



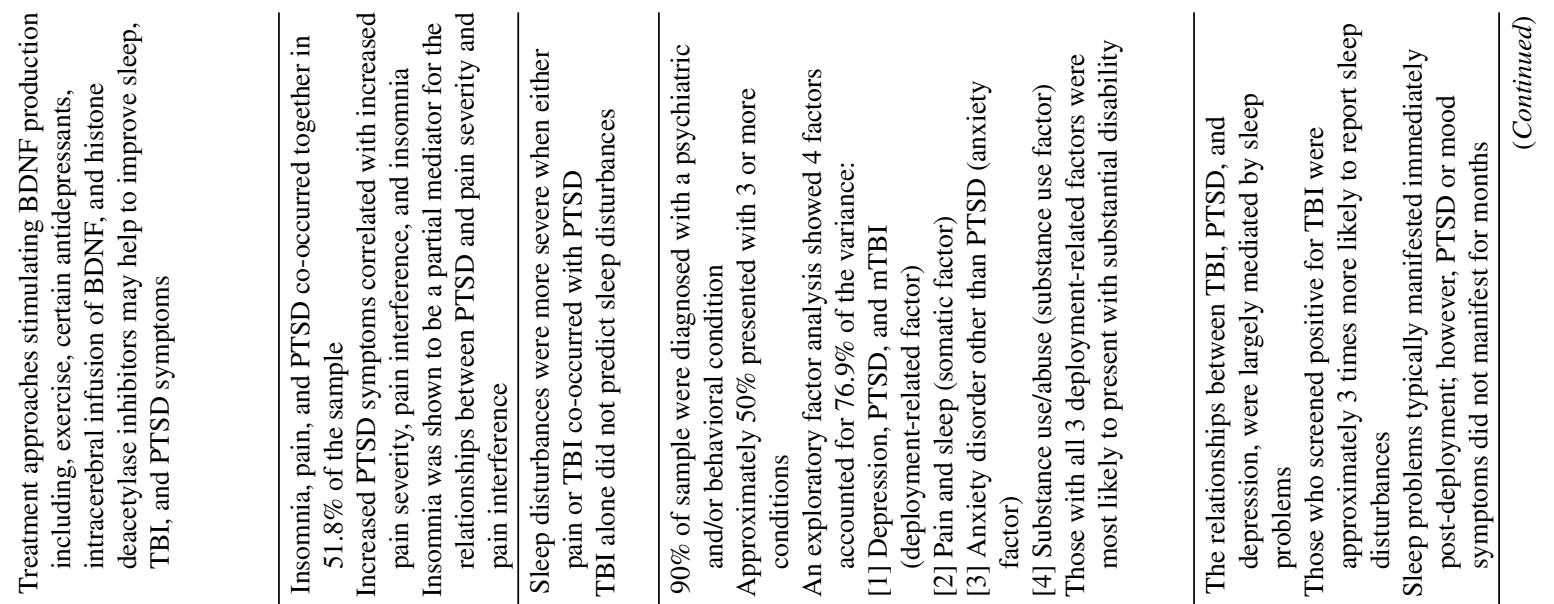

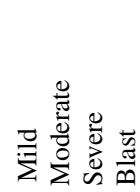

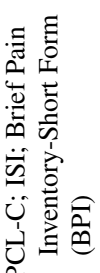

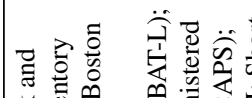

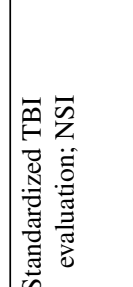

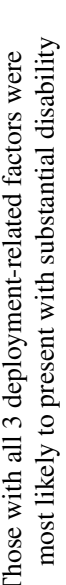

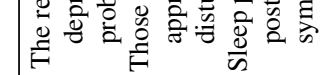

$\overleftrightarrow{z}$

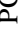

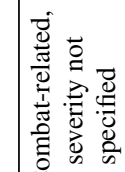

$\overleftarrow{z}$

$\overleftarrow{z}$

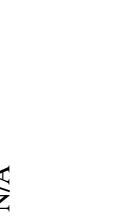

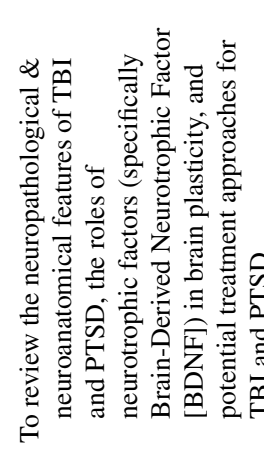

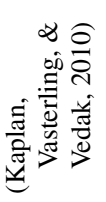

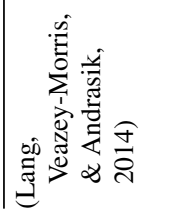

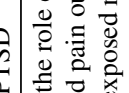

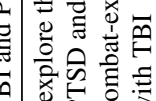

管 


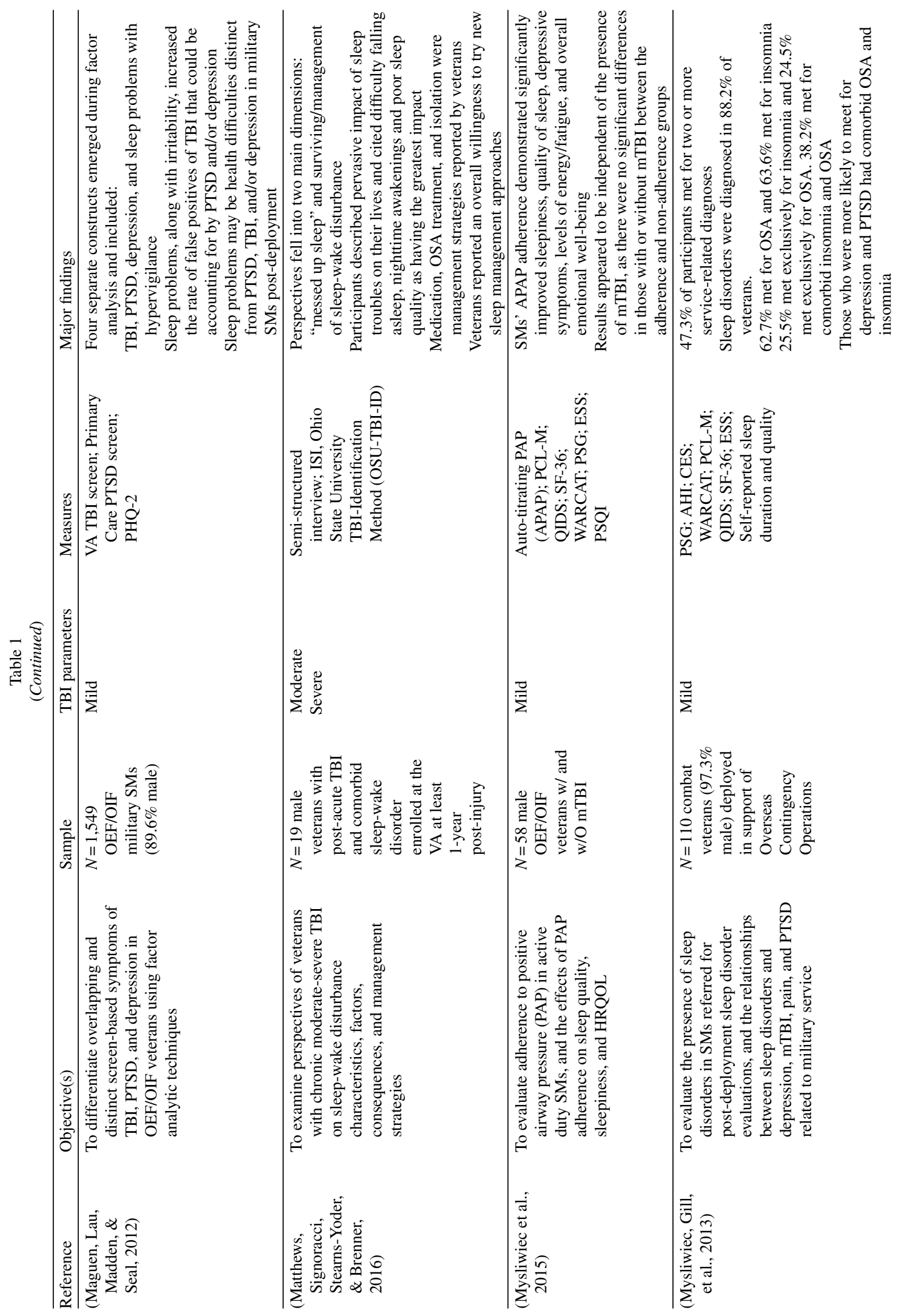



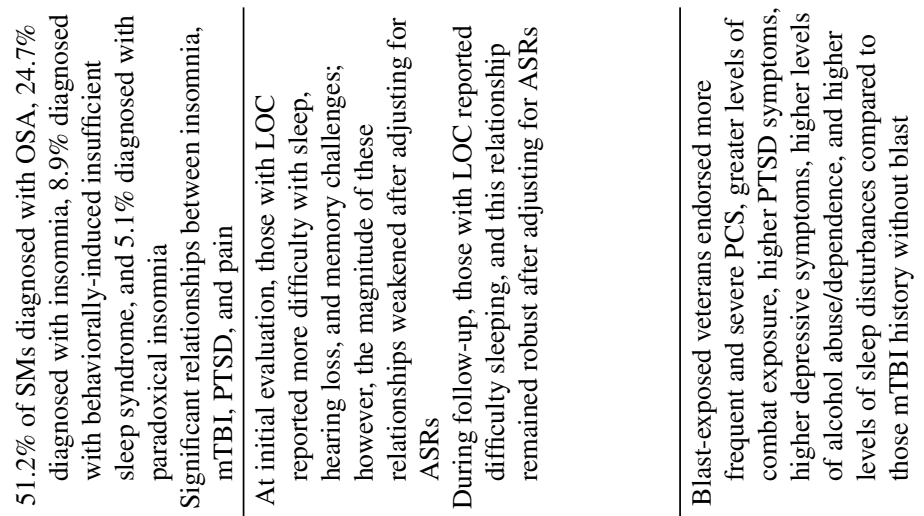

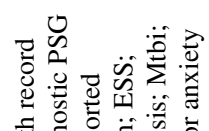

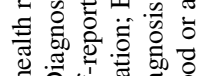

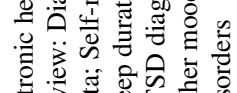

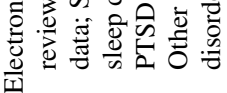

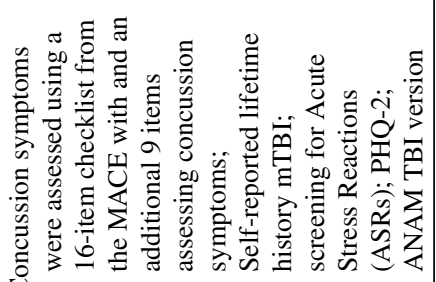

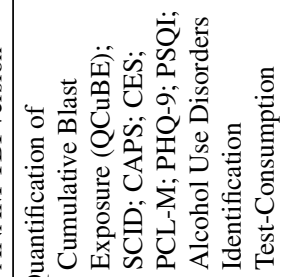

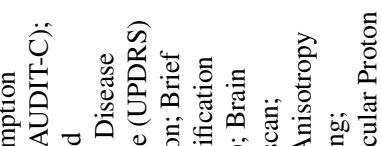
总

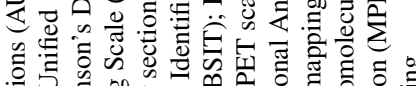

\begin{tabular}{|c|c|c|}
\hline & & \\
\hline$\frac{\pi}{z}$ & 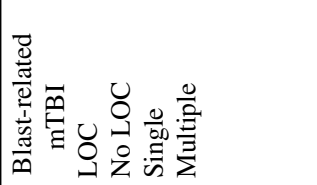 & 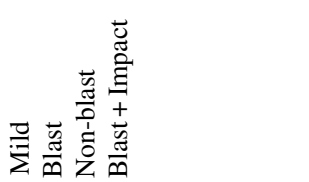 \\
\hline 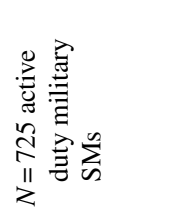 & 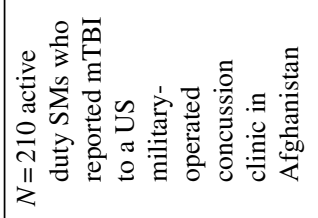 & 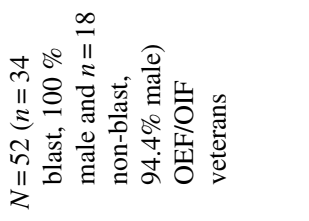 \\
\hline 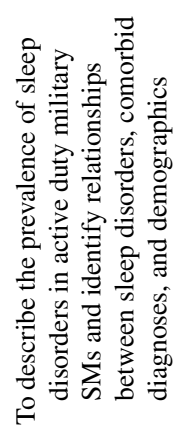 & 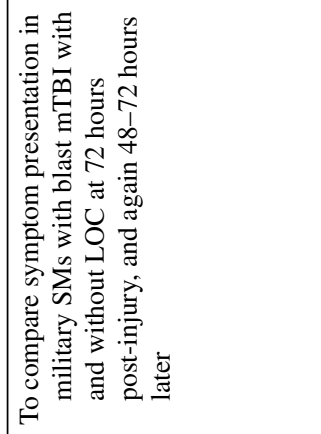 & 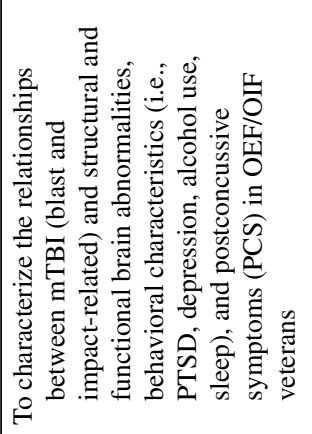 \\
\hline 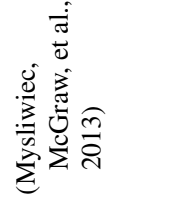 & 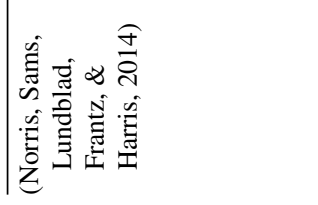 & 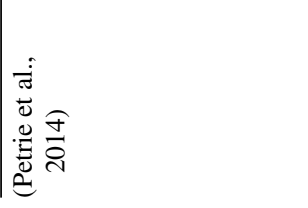 \\
\hline
\end{tabular}




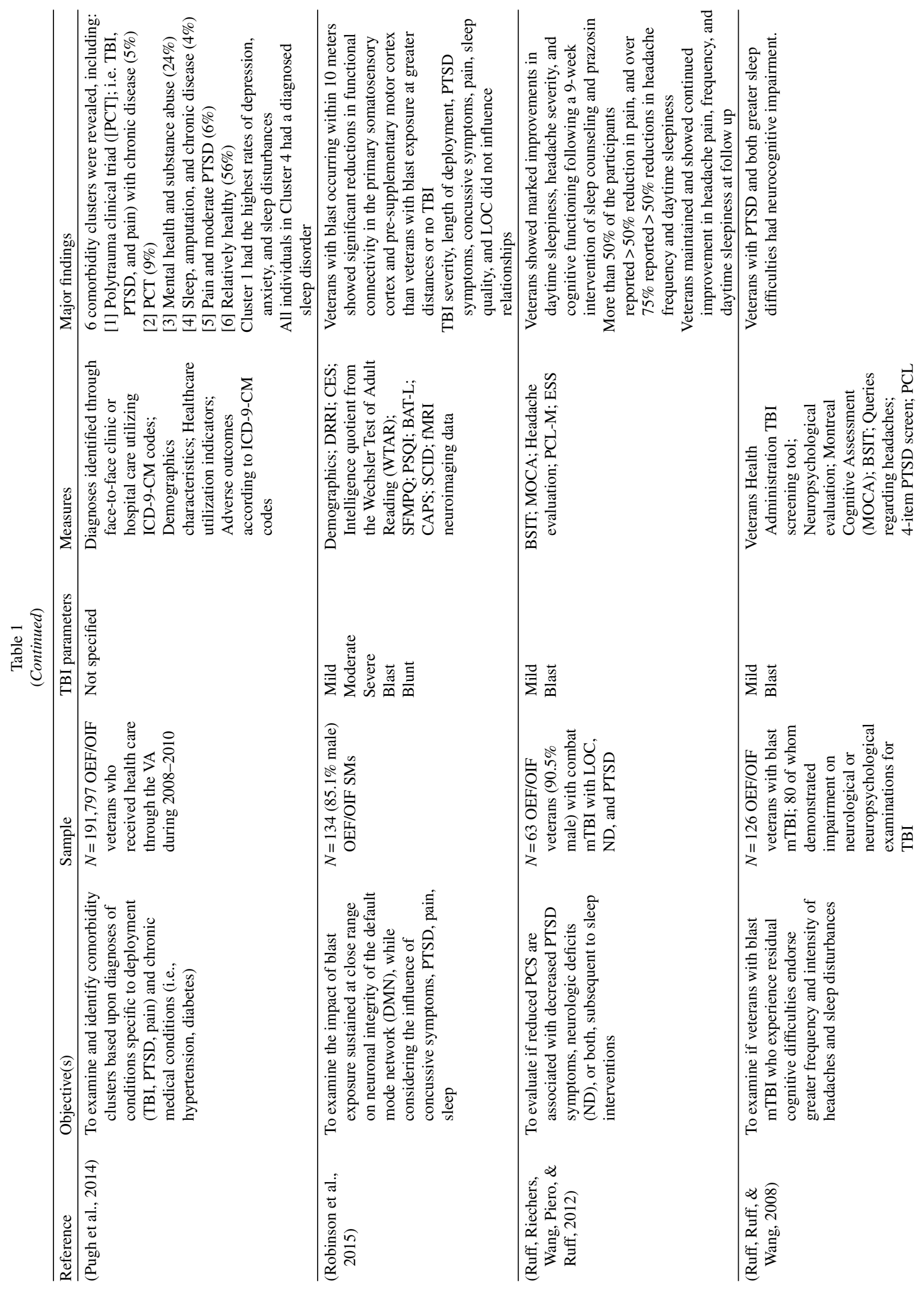



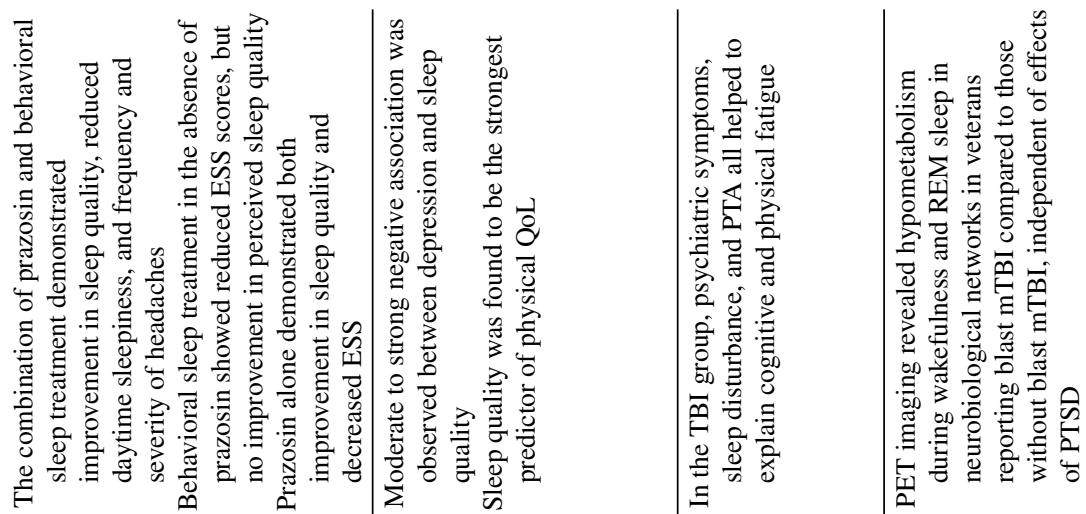

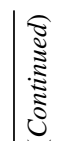
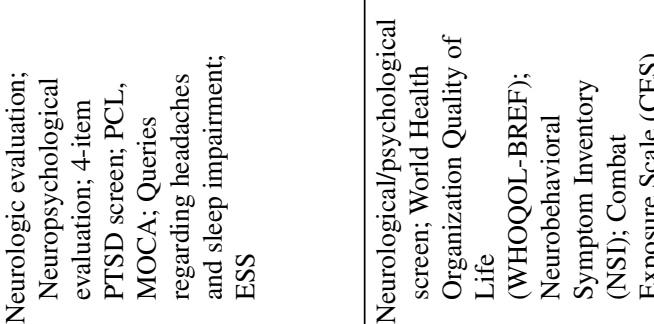

䄈咅

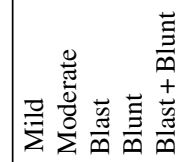

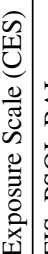

$\ddot{\Sigma}$

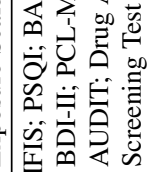

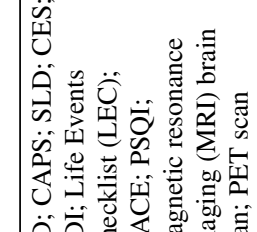

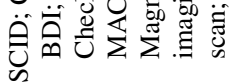

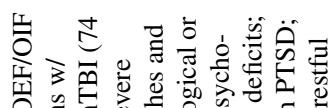

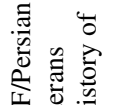

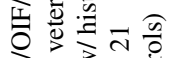

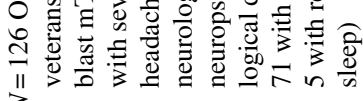

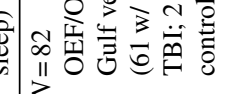

$\sum$

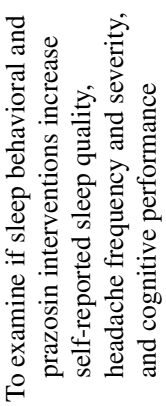

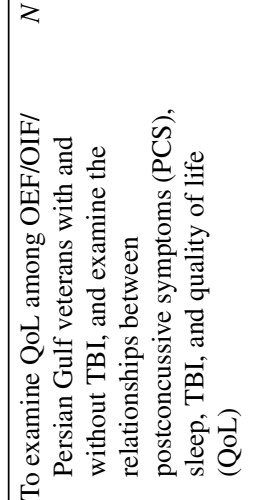

을

吾 $\frac{\vec{a}}{m}$

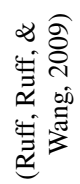
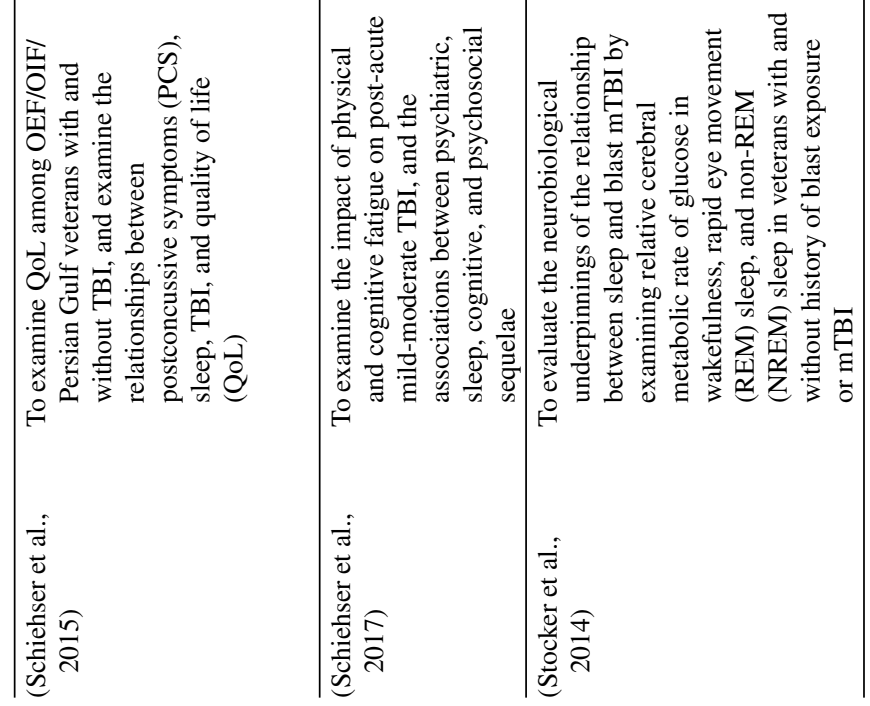


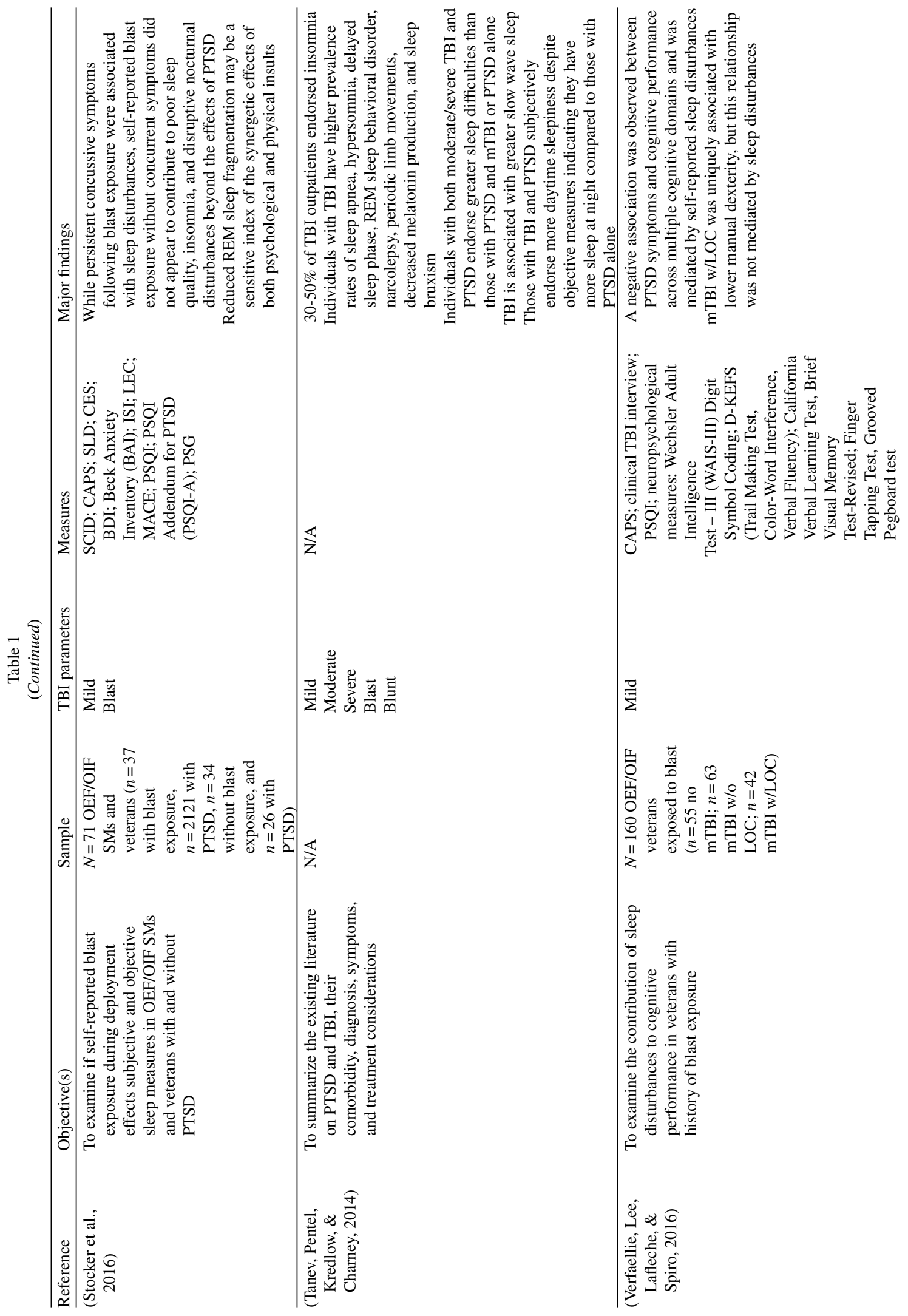



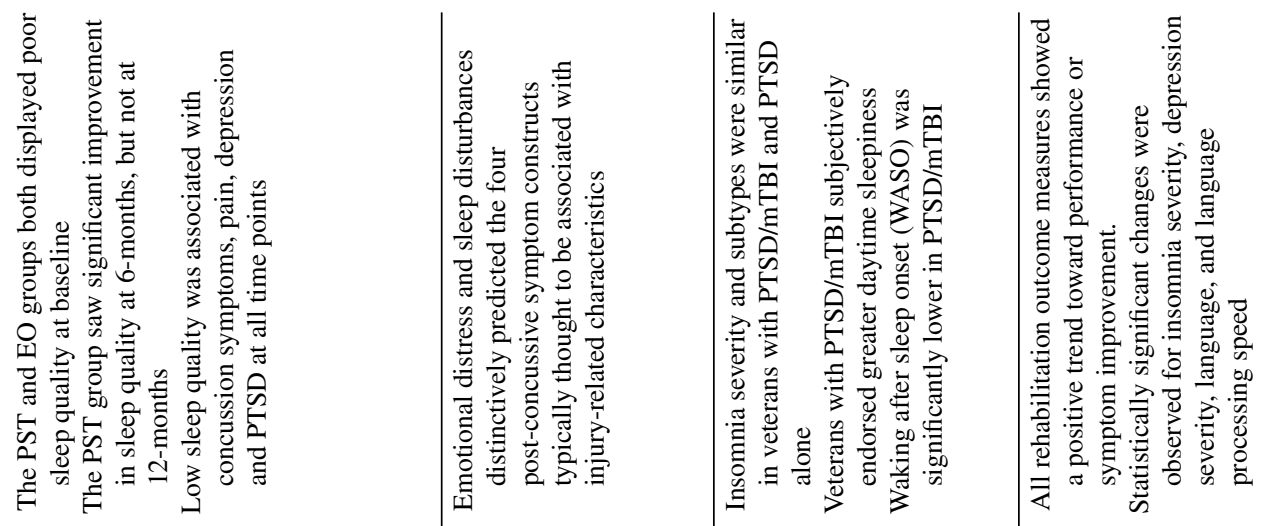

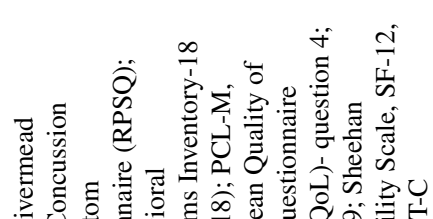

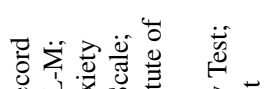

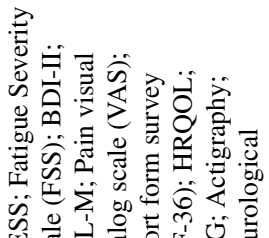

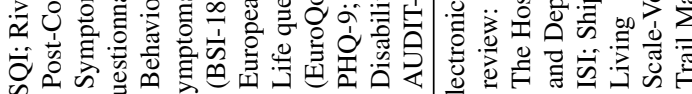

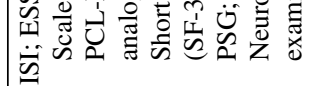
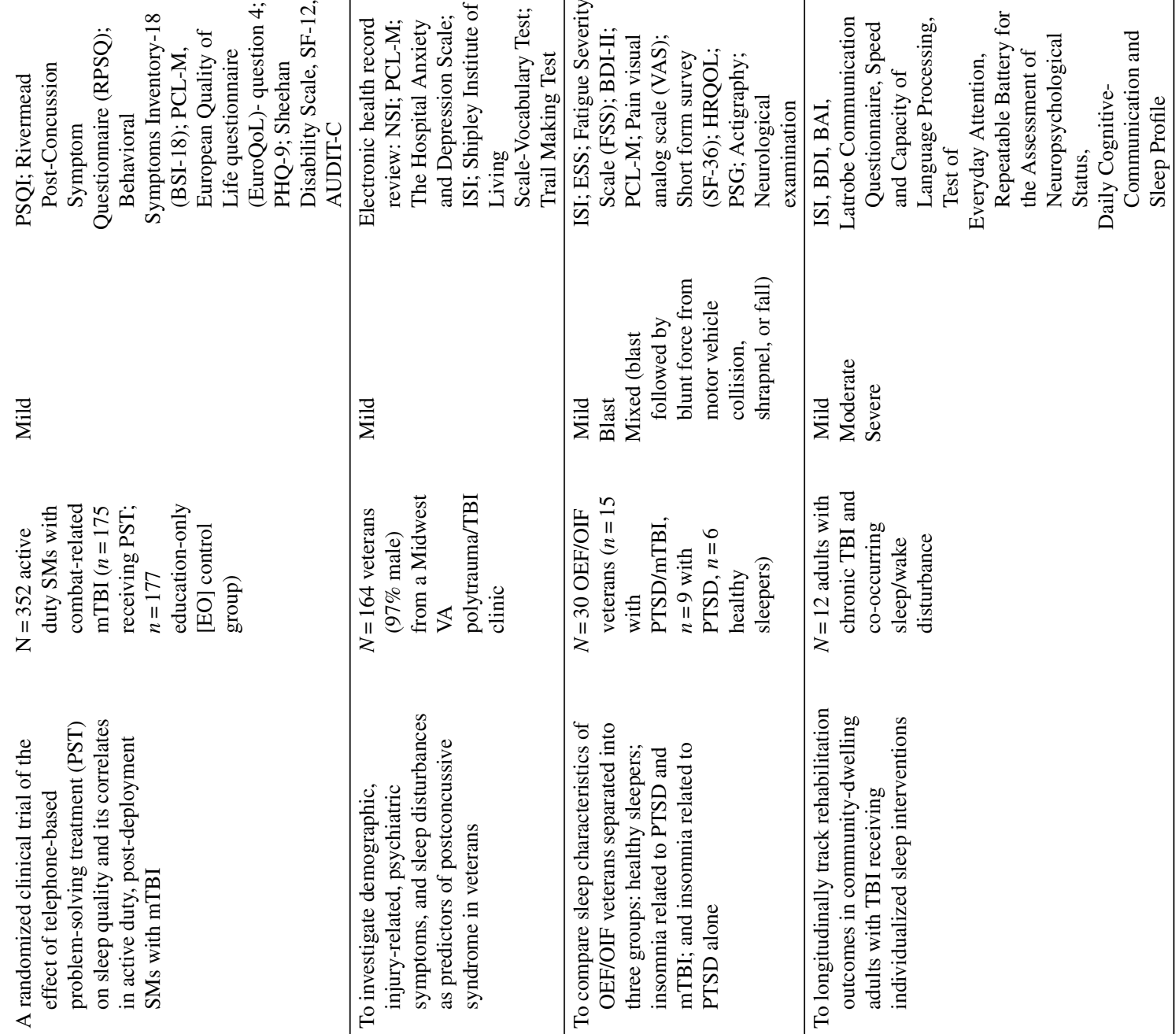

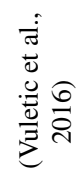
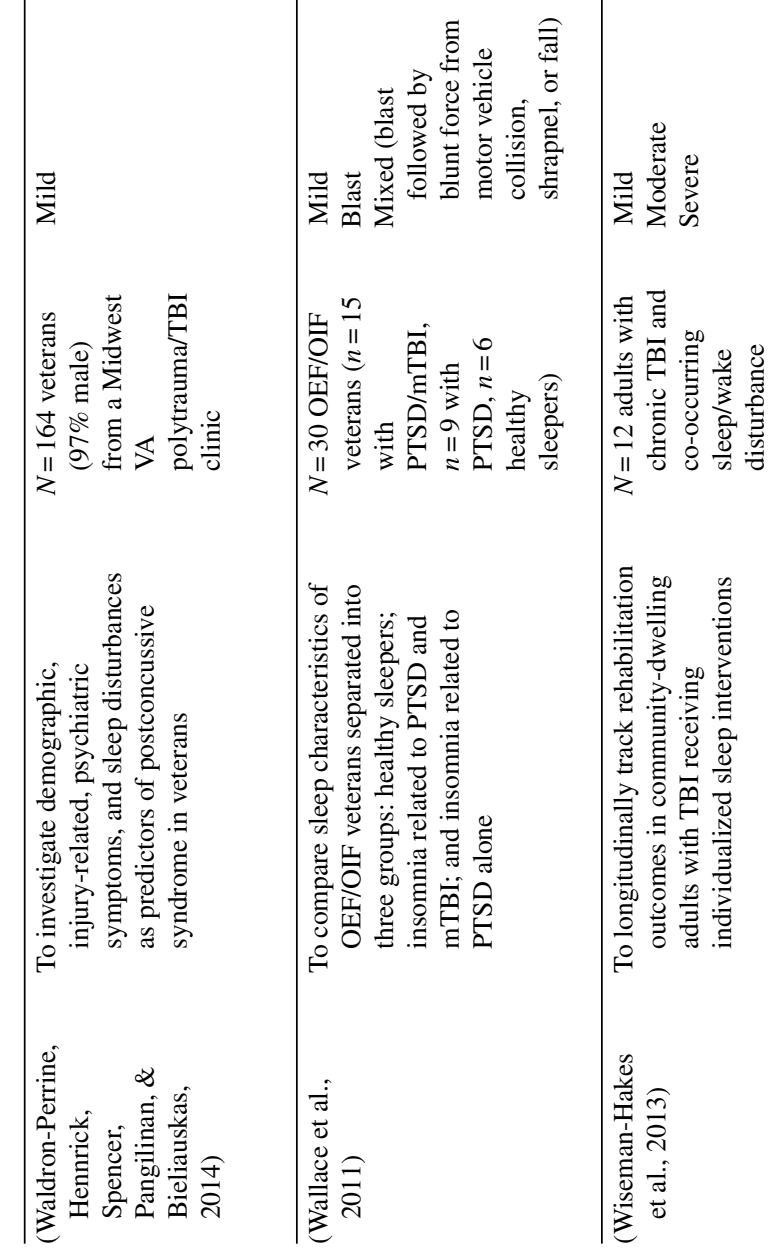
and TBI is SMS or veterans. Only one study to date at the time of this publication assessed sleep in the acute phase following TBI. Norris showed that TBI with loss of consciousness (LOC) was associated with greater self-reported difficulty sleeping compared to TBI without LOC in 210 active duty SMs who reported sustaining an mTBI to a US military-operated concussion clinic in Afghanistan. The greatest self-reported sleep difficulty associated with LOC was endorsed at 72 hours post-injury and again 48-72 hours after the initial TBI assessment (Norris, Sams, Lundblad, Frantz, \& Harris, 2014). These findings suggest that the onset of sleep disturbance can occur immediately following the point of injury and are strongly associated with LOC.

The Armed Forces Health Surveillance Center ([AFHSC]; 2013) reviewed the electronic medical records of 821,611 SMs with TBI at 3- and 12months post injury from a 13-year surveillance period examining 14 common TBI-related symptoms or conditions. Severity of sleep disturbance had the second greatest increase from three to 12 months across all levels of TBI severity when compared to a nonTBI control group. Other highly prevalent symptoms included headache, alcohol and substance abuse, and PTSD - all sequelae or comorbidities of TBI with known links to disrupted sleep. (AFHSC, 2013).

\subsection{Prevalence \& characterization of sleep disturbances in TBI}

Three studies focused primarily on estimating the prevalence of sleep disturbances in SMs and veterans with combat-related TBI using a wide variety of assessment tools including, self-report subjective and objective questionnaires, medical records review, objective sleep measurement (e.g., actigraphy, polysomnography (PSG)) and clinical interviews conducted in different settings (e.g., sleep clinics, laboratories). In a study by Collen et al (2012), one-hundred sixteen OEF/OIF combatexposed active duty SMs with documented mild to moderate TBI underwent a comprehensive sleep evaluation, including PSG and subjective assessments of sleep quality and daytime somnolence in a hospital sleep medicine clinic. Almost all (97.4\%) SMs experienced sleep disturbances, with the most common being hypersomnia, sleep fragmentation, insomnia, and OSAS (Collen et al., 2012). Using a large self-report battery of health assessments, Gill and colleagues estimated that one-third of 110 combat-exposed SMs with diagnosed sleep disorders and sleep disturbances also met criteria for two or more service-related disorders (Gill et al., 2014). The most commonly reported sleep disturbances were insomnia and OSA. SMs with sleep disturbances were divided into two groups: low-comorbidity (0-1 comorbid conditions) and high-comorbidity (2-3 comorbid conditions). SMs in the high comorbidity group were significantly more likely to be diagnosed with PTSD, depression, and/or TBI symptoms compared to SMs in the low comorbidity group. In combat-exposed active duty SMs, the percentage of soldiers endorsing two or more service-related sleep disturbances has been reported to be as high as $47.3 \%$ (Mysliwiec, Gill, et al., 2013; Mysliwiec, McGraw, et al., 2013).

Capaldi and colleagues isolated sleep features present in 69 active duty combat-exposed veterans with TBI and sleep disturbances through an electronic medical record review (including PSG, OSAS, and daytime sleepiness data) and found that frequency of trouble sleeping, number of nighttime awakenings, and daytime sleepiness were elevated across all TBI injury severity groups (Capaldi, Guerrero, \& Killgore, 2011). Compared to SMs without TBI, SMs with TBI are three times more likely to report sleep disturbances immediately following deployment (Macera, Aralis, Rauh, \& MacGregor, 2013). Focusing on more chronic, post-deployment sleep disturbances, Tanev and colleagues reported that veterans with TBI history had higher rates of OSAS, hyposomnia, REM sleep behavior disorder, narcolepsy, periodic limbic movements, sleep bruxism and increased slow wave sleep relative to veterans without TBI (Tanev, Pentel, Kredlow, \& Charney, 2014).

The variable prevalence estimates reported across studies of SMs and Veterans with TBI are likely a reflection of different assessment methods (e.g., PSG vs. self-report questionnaires) and a bias that may be introduced by studying patients in different settings (e.g., sleep clinics vs. general survey).

\subsection{Influence of TBI-related factors on sleep disturbances}

Eight studies suggested that injury-related factors may influence the nature and trajectory of sleep disturbances in SMs and veterans, as well as heath factors and functional impairment.

The mechanism of injury leading to TBI is a primary factor that may influence sleep disturbances in military samples. In the sample previously referenced by Collen and colleagues, participants with blast TBI 
reported significantly worse insomnia, whereas those with blunt TBI had greater levels of OSAS (Collen et al., 2012). Petrie et al. showed that when compared to veterans with TBI history without blast exposure, veterans with TBI history and blast exposure endorsed higher levels of sleep disturbances overall (Petrie et al., 2014). Furthermore, blast exposure followed by concussive symptoms has been associated with sleep disturbances, but this relationship was not found in those exposed to blast without current symptoms. These findings suggest that both blast- and blunt-TBI may increase risk for specific sleep disturbances, and that blast exposure may be especially important to the development of sleep disturbances in military samples, especially when concussive symptoms follow the initial injury.

TBI severity may also impact the severity of sleep disturbances and injury prognosis. In one of the few studies measuring sleep disturbances longitudinally after TBI, the AFHSC used electronic medical records to track changes in sleep disturbances change from three to 12 months post injury in SMs and veterans. Results showed that severe TBI was associated with the greatest increase in occurrence of new sleep disorders at 12 months relative to mild and moderate TBI, those with prior TBI, and SMs and veterans with no current symptoms (AFHSC, 2013).

LOC has also shown to be significantly associated with more sleep disturbances, in particular nightmares, in those with TBI compared to those with TBI without LOC (Farrell-Carnahan et al., 2013). As detailed above, Norris et al. also found that LOC at time of injury was associated with more severe sleep disturbances (Norris et al., 2014).

The number of TBIs may also be of particular importance when estimating the prevalence of sleep disturbances in military samples with TBI. Bryan assessed the prevalence of clinical insomnia across TBI frequency and found compared to those with zero TBIs, four times as many participants with one TBI and 10 times as many participants with multiple TBIs met for clinical insomnia (Bryan, 2013). These results remained robust when controlling for PTSD, depressive symptoms, and post-concussive symptoms.

Finally, the presence or absence of prior TBI may also impact sleep disturbances in TBI. In a longitudinal study of the relationship between TBI status and sleep disturbances, Holster et al. found that Air Force veterans with a pre-deployment history of TBI endorsed more insomnia symptoms at baseline when compared to those without prior TBI history (Holster, Bryan, Heron, \& Seegmiller, 2017). In turn, baseline insomnia symptoms were associated with increased risk for sustaining a TBI during deployment. Chronic insomnia at baseline was also associated with both pre-deployment TBI history and TBI during deployment. It is important to note that differences have been observed in neurological changes between military SMs with blast exposure occurring both within and greater than 10 meters that were not influenced by sleep quality (Robinson et al., 2015).

Of note, none of the articles reviewed accounted for the timing of injury occurrences in those with more than one TBI. This is likely a critically important factor to consider when assessing the impact of TBI frequency and prior TBI on sleep disturbances. More specifically, it is important to consider the timing between the injuries themselves. When full recovery from one injury does not preceded the onset of a second injury, symptoms are likely to exacerbate and become pervasive. Thus, summative TBIs may result in unique profiles of sleep disturbances that influence treatment effectiveness and outcomes.

Together, the findings to date unequivocally establish a strong relationship between TBI and sleep disturbances in SMs and veterans. Furthermore, a number of factors related to injury etiology may modulate or mediate this relationship but remain underexplored. Larger samples and more comprehensive study designs are needed to further characterize this relationship and determine whether assessment and treatments strategies should be informed by these factors. Recognizing the challenges of conducting longitudinal studies, access to medical records would provide a more efficient and cost-effective study design for better determining estimates of time between injury and symptom onset.

\subsection{Interactions between sleep disturbances, $T B I$, and comorbid conditions}

Sleep disorders and TBI are complex and heterogeneous conditions with shared symptomology that and are both independently and jointly associated with psychiatric and medical comorbidities. Twenty-four of the studies included in this review reported on comorbid psychiatric and medical conditions of TBI. For instance, Gill et al. showed severity of PTSD, sleep disturbances, and depressive symptoms increase as the number of comorbid conditions increase in SMs with TBI (Gill et al., 2014). The combination of these conditions complicates assessment and management strategies and uniquely impacts functional outcomes. TBI resulting 
from combat exposure has been shown to most commonly co-present with psychological trauma-related disorders (i.e. PTSD), chronic pain, and insomnia in over $51 \%$ of veterans (Lang, Veazey-Morris, \& Andrasik, 2014). The tight relationship between TBI, sleep disturbance and psychiatric conditions has risen the possibility that sleep may serve as a common factor linking TBI with its comorbidities, which in tune may suggest a summative effect of co-occurring conditions and TBI on sleep disturbances relative to TBI alone. Lew and colleagues observed that sleep problems were more severe when PTSD was comorbid with TBI compared to those with TBI alone (Lew et al., 2010).

Furthermore, Macera et al. observed that veterans who screened positive for TBI and PTSD were 3-times more likely to report sleep problems compared to TBI alone. This suggests that the relationship between TBI and its associated comorbidities may even be moderated or mediated by sleep disturbances (Macera et al., 2013). This finding was supported in two studies by Verfaellie et al. and Waldron-Perrine that each reported sleep disturbances mediated the relationships between emotional distress, cognitive functioning, and self-reported post concussive symptoms more so than TBI alone (Verfaellie, Lee, Lafleche, \& Spiro, 2016; Waldron-Perrine, Hennrick, Spencer, Pangilinan, \& Bieliauskas, 2014). In one of the few studies on pre-TBI sleep factors, Elliott et al. showed that the resiliency personality phenotype predicted less sleep troubles in military samples who go on to develop TBI when compared to other phenotypes (Elliott et al., 2017). This finding suggests that pre-injury factors may play a role in the course of TBI and provides support for future research examining pre-injury sleep factors in military SMs and veterans prior to deployment. A greater understanding of the role of sleep in the trajectory of TBI may yield novel, modifiable sleep-related targets for preventive efforts or engagement through sleep-focused, individualized interventions aimed at mitigating symptoms and restoring function through improved sleep.

Stocker and colleagues examined positron emission tomography (PET) scans of OEF/OIF veterans with and without comorbid blast TBI and PTSD and found that there was a detectable decrease of regional cerebral metabolic rate of glucose during REM sleep and wakefulness among veterans with prior blast TBI than those without blast TBI history when adjusting for any effects of PTSD (Stocker et al., 2014). It was suggested that the areas of glucose hypometabolism, including the right basal ganglia, amygdala, hippocampus and parahippocampal gyrus, culmen, associative visual cortices and medial frontal cortices may be susceptible to long-term effects of blast TBI, including chronic concussive symptoms and/or maladaptive responses to stress (Stocker et al., 2014). A separate study examining objective sleep features in a comorbid TBI/PTSD sample from Stocker and colleagues revealed that REM sleep fragmentation may be a potentially sensitive index to the synergistic effects of TBI and PTSD, as the only significant interaction effect between TBI and PTSD was observed in the amount of REM sleep fragmentation (Stocker et al., 2016). A summative effect of comorbid TBI on sleep problems has also been observed in clinical settings, where individuals at a polytrauma clinic had the highest rates of sleep disturbance, anxiety, and depression (Pugh et al., 2014).

Wallace and colleagues showed that insomnia severity was not significantly different between those with PTSD/TBI and PTSD alone (Robinson et al., 2015; Wallace et al., 2011). These results do contrast the literature; however, it is likely that the difference in findings is due to the heterogeneity of the sample when using sleep quality as an outcome or other factors unique to neurological changes.

Finally, those with comorbid TBI and PTSD report more daytime sleepiness despite objective measures indicating they have greater total sleep time relative to those with TBI alone (Tanev et al., 2014). This finding was replicated in a study by Wallace et al (Wallace et al., 2011) and highlights the importance of using multimodal data collection methods to best understand the complex relationships between TBI and its common comorbidities and sleep disturbances. Self-reported sleep disturbances provide rich subjective data, that when taken in combination with objective sleep measures is likely to better support optimal intervention selection compared to intervention selection based solely from objective data sources.

An enhanced understanding of how sleep disturbances present in TBI when comorbidities are present and how the sleep profiles of comorbid groups compare to those with TBI alone is needed to improve assessment and guide optimal courses for intervention (Lang et al., 2014). This line of research is especially important considering around $50 \%$ of SMs and veterans experience three or more deploymentrelated conditions, and that those with three or more deployment-related conditions are more likely to present with substantial disability relative to those with TBI alone (Lippa et al., 2015). Nevertheless, the 
literature to date highlights that more severe sleep disturbances occur when TBI presents with co-morbid conditions.

\subsection{Sleep disturbances: Distinct domain or secondary condition?}

Findings from two studies using factor analysis suggest a TBI diagnosis presents with both overlapping and distinct symptom clusters with its most common comorbid conditions (e.g., PTSD), and even highlights sleep disturbances as a distinct cluster that transcends diagnostic categories (Jaramillo et al., 2015; Maguen, Lau, Madden, \& Seal, 2012).

These studies identified distinct symptom clusters (i.e. low/high distress) across larger diagnostic groups that may improve the ability to differentiate SMs experiencing comorbid conditions with shared symptomology. While these studies chose to cluster on symptoms and not diagnoses, results did show some clusters were more likely than others to contain a particular diagnosis. For example, a 'moderate/high distress' cluster represented by headache and memory impairment capturing a high rate of those with TBI (Jaramillo et al., 2015) or a high rate of those with PTSD being represented in a 'high distress' or 'pain and behavioral health' cluster represented by anxiety and substance abuse (Jaramillo et al., 2015). Maguen and colleagues used factor analysis to differentiate distinct from overlapping screening-based symptoms in 1549 veterans with TBI, PTSD, and depression from postdeployment screening data (Maguen et al., 2012). Four constructs merged, three of which were represented by the diagnoses themselves (i.e. PTSD, TBI, depression) and an intriguing $4^{\text {th }}$ construct: "hypervigilance and sleep problems" (Maguen et al., 2012). The findings from Maguen's study show symptoms unique to TBI that may help improve assessment and targeted treatments when attempting to separate TBI from its comorbid conditions. Arguably, the most notable finding is the evidence for a distinct 'hypervigilance/sleep disturbance' cluster that highlights the needs to further examine hyperviligence and disrupted sleep symptoms as a unique, combined factor influencing post-deployment health in military groups with TBI. Overall, the findings from studies using factor analysis suggest that while the co-occurring diagnoses themselves may be difficult to differentiate, distinctive phenotypes may exist that can aid more optimal assessment and selection of treatment targets.
Pugh et al., and Lippa et al., both showed that although disrupted sleep was distinct from both TBI and PTSD, TBI and PTSD failed to be different from one another across sleep variables (Lippa et al., 2015; Pugh et al., 2014). Capaldi and colleagues also examined the specificity of sleep disturbances related to TBI and PTSD in 69 active duty combat veterans and found that PSG failed for the most part to significantly differentiate TBI from PTSD (Capaldi et al., 2011). However, those with PTSD did have a higher frequency of nighttime arousals and those with TBI had more slow wave sleep. This finding may be reflective of arousal brain states linked to sleep in those with PTSD (Germain, Buysse, \& Nofzinger, 2008) theories supporting slow wave sleep as a critical component for recovery after TBI (Shekleton et al., 2010).

\subsection{Sleep-focused interventions may improve TBI symptoms}

TBI interventions rarely include sleep-specific assessments and strategies, and few studies have used sleep-focused interventions to assess TBI symptom change and improvements in functional outcomes in military SMs and veterans (Gilbert et al., 2015).

As previously highlighted, OSA is a prevalent sleep disorder in SMs and veterans with TBI. Barr et al reported over $50 \%$ of recently deployed military SMs with TBI endorsed more restorative sleep and improved health-related quality of life (HRQoL) of life at 12 months following provision of standard care, which included continuous positive airway pressure (C-PAP) therapy and/or cognitive behavioral therapy (Barr et al., 2015). Additionally, those with TBI who experienced improved sleep post-treatment also saw a reduction of PTSD and depression symptoms at 3-months. Mysliwiec et al also showed reduced daytime sleepiness and improved sleep quality in 58 male military personnel with and without TBI following autotitrating positive airway pressure (APAP) (Mysliwiec et al., 2013). Participants who improved used APAP for four hours or more a night for at least 70 percent of all nights during a consecutive one-month period. Participants in the improved sleep group also experienced functional improvement related to depressive symptoms, energy/fatigue, and emotional well-being. Although these two studies used a treatment specific to OSAS, findings support the modifiable nature of sleep in TBI and show the potential for using sleep-focused interventions for improving not only sleep, but also health and functioning. 
Ruff et al. used a 9-week combination treatment consisting of a behavioral intervention (i.e. sleep hygiene counseling) and prazosin in 126 veterans with blast mTBI to assess change in disrupted sleep and daytime sleepiness (Ruff, Ruff, \& Wang, 2009). Disrupted sleep was assessed through two questions on whether or not participants experienced impaired sleep and what they believed to be the nature of their sleep impairment and daytime sleepiness was measured by the Epworth Sleepiness Scale (ESS). At post-treatment, those in the combination treatment group reported, on average, improved self-report sleep quality and decreased or complete remission of nightmares. Furthermore, 100 percent of participants saw a significant reduction in daytime sleepiness. A second study by Ruff and colleagues using a 9-week combination behavioral-pharmacological treatment of sleep counseling and prazosin in 63 veterans with combat mTBI with LOC saw a greater than 50 percent post-treatment reduction in daytime sleepiness in over $78 \%$ of participants, as measured by the ESS (Ruff, Riechers, Wang, Piero, \& Ruff, 2012). This greater than $50 \%$ reduction in daytime sleepiness was maintained and improved upon (78\% to $89 \%$ ) at a 6 month follow up. It is important to note that this study also found improved outcomes with behavioral and pharmacological interventions alone, which is evidence of the potential relative utility of these approaches.

Finally, in the only randomized clinical trial on sleep disturbances and TBI known at date, Vuletic et al tested the effectiveness of a 6-month telephonebased problem-solving treatment (PST) intervention for improving sleep quality in 356 active duty SMs with TBI and poor baseline sleep quality (Vuletic et al., 2016). The PST intervention involved 12 biweekly phone calls addressing participated-selected issues related to their sleep. Results showed that when compared to an education only (i.e., educational brochures on sleep) control group, the PST group saw improved sleep quality at 6 months, but not at 12 months, as measured by the Pittsburgh Sleep Quality Index (PSQI). This finding suggests PST may be an effective treatment to improve sleep acutely in military cohorts, but more work needs to be done to determine the long-term effectiveness of this approach.

In a community-based, single-blind longitudinal study, Wiseman-Hakes and colleagues examined objective and self-report cognitive and emotional rehabilitation outcomes in adults with TBI and comorbid sleep/wake disturbances (2013). This study used individualized sleep/wake disorder treatment, which included sleep hygiene, pharmacological intervention, and sleep apnea interventions. Statistically significant post-intervention changes in mood, performance, and cognitive symptoms were observed-suggesting sleep-focused treatments may help to improve rehabilitation outcomes in those with TBI across all levels of severity.

When examining the perspectives of 19 male veterans with post-acute TBI and comorbid sleep-wake disorder enrolled at the VA at least 1-year postinjury using semi-structured sleep-related interviews, Matthews and colleagues qualitatively extrapolated two perspective dimensions based on qualitative analysis of responses: "messed up sleep" and surviving/management of sleep disturbances (Matthews, Signoracci, Stearns-Yoder, \& Brenner, 2016). This sample of veterans also reported that their sleep troubles had a significant negative impact on daily living. Specifically, difficulty falling asleep, nighttime awakenings, and poor sleep quality were cited as having the greatest adverse impact on overall daytime functioning. This study suggests veterans recognize the challenging nature of managing sleep disturbances and that sleep problems can be difficult to manage.

Findings from these studies provide preliminary support for the effectiveness of sleep interventions to improve not only sleep symptoms, but also health and functioning in military samples with TBI. These studies suggest that both sleep-focused pharmacological and behavioral interventions may be efficacious for improving outcomes for SMs and veterans with TBI either individually or in combination. Albeit promising, these studies warrant confirmatory controlled effectiveness trials. It is important to note than only a scarce body of literature to date has looked at how sleep interventions may improve TBI symptoms in military SMs and veterans (Ruff et al., 2012) — another underexplored area to guide future research efforts.

\section{Discussion}

The purpose of this article was to conduct a scoping review to synthesize the current science on disrupted sleep and TBI as it pertains to combat-exposed military SMs and veterans. The 41 articles included in this review were published between 2008-2018 inclusively and were on military samples predominantly from the OEF/OIF/OND conflicts, with some studies including Persian Gulf War veterans. 
Most of the literature to date has focused on the establishing the prevalence of sleep disturbances in combat- TBI and the identification of unique sleep profiles between military SMs and veterans with and without TBI, using a variety of sampling methods and sleep measurement methods. Eight of the studies reviewed examined the influence of TBI-related factors on sleep and co-occurring psychiatric and medical conditions, such as PTSD. Further, two studies reviewed suggested that sleep symptoms may help to distinguish TBI from its co-occurring conditions, but results remain inconclusive as to which symptoms provide the best utility demarcating TBI from co-occurring conditions with shared symptomology. The majority of studies reviewed were descriptive and cross-sectional in nature, with few testing the effectiveness of interventions and measuring change in symptomology over time. This review demonstrated there is a rich and diverse existing foundational literature on sleep profiles and influencing health and injury factors in military samples with TBI for researchers can build upon in future research. This review also sheds light on the work yet to be done measuring pre-TBI or pre-deployment sleep symptoms that may infer TBI risk, determining prodromes for the development of sleep disturbances after TBI, sleep-related mechanistic processes that influence symptoms and functional outcomes after TBI, and the long-term effects of chronic sleep disruption in TBI on functioning, health, and quality of life. There is a clear need for evidence-based, systematic research developing and testing sleep-related interventions aimed and improving both sleep and TBI symptoms and long-term functional and health outcomes.

\subsection{Clinical and research implications}

Historically, disturbed sleep has been viewed as a symptom of TBI. This implies a temporal relationship where TBI onset precedes the development of sleep symptoms. Much of the literature in this review examined the co-occurring nature of sleep disturbances and TBI or disturbed sleep as a mediator or moderator in the relationship between TBI and poor outcomes in combat-exposed military samples. Furthermore, it is known that sleep disturbances are associated with reduced overall functioning in military samples with TBI (Ettenhofer, Melrose, Delawalla, Castellon, \& Okonek, 2012) and that improving sleep can lead to TBI symptom reduction and increased HRQoL (Barr et al., 2015). Thus, disrupted sleep likely plays a much greater role in the course of TBI-one where sleep disturbances and TBI share bidirectional and even cyclical relationship (Gilbert et al., 2015). Future research efforts are needed to examine the full trajectory of sleep disturbances and TBI in military groups.

Although the current state of the field is keenly attuned to sleep disturbances profiles following TBI, research examining TBIs sustained among individuals with preexisting sleep disturbances remains scarce. There is even less research related to the examination of whether sleep/wake difficulties can be conceptualized as presenting independently of TBI rather than as a symptom of TBI. Although disturbance in normative sleep may be a manifestation of the neurobiological changes of TBI in civilians and veterans alike, it is possible for that for military SMs, the nature of military service, specifically while deployed, may give rise to disrupted sleep patterns prior to sustaining a TBI-an area yet to be studied. This is an intriguing line of research to explore not only due to the ability to demonstrate sleep disturbances as a risk factor for injury to the head, but given the existing literature linking inadequate sleep to increased risk for physical injuries it also warrants examining the effect of disrupted sleep on other physical injuries while in the field. Further, it could be argued that just as sleep disturbances can develop during deployment in those who do not sustain TBI, sleep disturbances could develop subsequent to a TBI and be better accounted for by the same factors as those without TBI than the TBI itself.

As our previous studies (Germain et al., 2014; Germain, Shear, Hall, \& Buysse, 2007) have demonstrated the effectiveness of brief behavioral sleep treatments in SMs and veterans with deploymentrelated comorbidities, behavioral sleep treatments offer a promising treatment approach. There is also a need to improve treatment approaches for OSA (to improve adherence), better extend trials on hypersomnia to military populations, and identify and test novel pharmacological agents for improving sleep in SMs that do not violate military drug policies. Research has shown that both military SMs and veterans are willing to try novel approaches to improving their sleep, which establishes this cohort as an engaged population (Matthews et al., 2016).

\subsection{Limitations}

Although this broad overview of the literature on TBI and sleep disturbances in military samples may 
lack the rigor that comes with a more exhaustive systematic review or even meta-analysis, this review was intended to gather a diverse representation of the research that has been conducted to date. This method allowed for visual inspection of any skewness in the current literature, which this review did demonstrate. This approach also allowed for the identification of knowledge gaps that will be critical to fill moving forward to advance the state of the science on combatrelated TBI and sleep disturbances. Scoping reviews are commonly implemented when the goal is to lay the foundation for a guide for when working with specific cohorts and when the extant literature would not be appropriate to summarize through systematic review (Peters et al., 2015). With that in mind, the nature of this review leads to the possibility that articles examining the topics of interest were missed. As such, the current review may not fully characterize the literature to date.

A challenge often presents when consolidating evidence from current bodies of literature is heterogeneity among the articles examined. This review of the literature resulted in diverse patterns across studies on operational definitions and inclusion/exclusion criteria (e.g., how TBI was defined or assessed), variables used to represent constructs of interest (e.g., the use of varying subjective or objective sleep parameters to measure the construct of 'poor sleep'), and the diversity of assessment tools used for measurement (e.g., semi-structured interviews, PSG, self-report scales). This heterogeneity becomes especially difficult to interpret when research lacks universally accepted terminology, operational definitions, and measurement tools for the constructs being examined. For example, differences in criteria used to identify the presence or absence of a TBI may create unintentional baseline differences between groups across studies that have a direct influence on outcomes independent of the variables measured. For example, two independent studies that established neuropsychological differences between military veterans with and with mTBI, but used different assessment batteries, makes it challenging for researchers to feel confident about the true effect. It is important to note that the issue of research methodological heterogeneity is not a challenge specific to military research, but in fact a widespread challenge the greater field of research is currently facing; and therefore, something researchers should be cognizant of when conducting their own studies. We advise that future research move towards implementing a more standardized methodology, which should include multi-modal assessment of sleep disturbances. Improved and comprehensive assessment methods will lay a strong and much needed foundation for more optimal selection of interventions and their potential for success. Finally, this review represents a bias towards articles that were published only in English speaking, peer-reviewed journals.

\section{Conclusion}

This scoping review synthesized the existing literature on sleep disturbances and TBI in military SMs and veterans and sheds light onto critical knowledge gaps that can guide future research aimed at answering the many remaining questions regarding the relationship between sleep disturbances and combat-related TBI. Our goal for this review was to add to the preexisting knowledge in the realm of TBI and sleep in SMs and military veterans. Moreover, it is our hope that this information can ultimately be utilized by researchers and clinicians to further expand the fields' awareness to and knowledge of the extant literature, while also encourage the advancement of novel and innovative research focused on prevention efforts, the treatment of, and long-term care for the unique population of military SMs and veterans with TBI and sleep disturbances.

\section{Conflict of interest}

None to report.

\section{References}

Armed Forces Health Surveillance Center (AFHSC). (2013). Incident diagnoses of common symptoms ("sequelae") following traumatic brain injury, active component, U.S. Armed Forces, 2000-2012 (2152-8217). Retrieved from

Babson, K. A., \& Feldner, M. T. (2010). Temporal relations between sleep problems and both traumatic event exposure and PTSD: A critical review of the empirical literature. J Anxiety Disord, 24(1), 1-15. doi:10.1016/j.janxdis.2009.08.002

Barr, T., Livingston, W., Guardado, P., Baxter, T., Mysliwiec, V., \& Gill, J. (2015). Chapter 8 Military Personnel With Traumatic Brain Injuries and Insomnia Have Reductions in PTSD and Improved Perceived Health Following Sleep Restoration: A Relationship Moderated by Inflammation. Annu Rev Nurs Res, 33, 249-266. doi:10.1891/0739-6686.33.249

Barshikar, S., \& Bell, K. R. (2017). Sleep Disturbance After TBI. Curr Neurol Neurosci Rep, 17(11), 87. doi:10.1007/s11910017-0792-4 
Bell, K. R., Bushnik, T., Dams-O'Connor, K., Goldin, Y., Jeanne, M. H., Lequerica, A. H., \& Zumsteg, J. M. (2018). Sleep after TBI: How the TBI model systems have advanced the field. NeuroRehabilitation, (Preprint), 1-10.

Bogdanov, S., Naismith, S., \& Lah, S. (2017). Sleep outcomes following sleep-hygiene-related interventions for individuals with traumatic brain injury: A systematic review. Brain Inj, 31(4), 422-433. doi:10.1080/02699052.2017.1282042

Bramoweth, A. D., \& Germain, A. (2013). Deployment-related insomnia in military personnel and veterans. Curr Psychiatry Rep, 15(10), 401. doi:10.1007/s11920-013-0401-4

Breen, A., Blankley, K., \& Fine, J. (2017). The efficacy of prazosin for the treatment of posttraumatic stress disorder nightmares in U.S. military veterans. J Am Assoc Nurse Pract, 29(2), 65-69. doi:10.1002/2327-6924.12432

Bryan, C. J. (2013). Repetitive traumatic brain injury (or concussion) increases severity of sleep disturbance among deployed military personnel. Sleep, 36(6), 941-946. doi:10.5665/sleep. 2730

Bryant, R. A., Creamer, M., O'Donnell, M., Silove, D., \& McFarlane, A. C. (2010). Sleep disturbance immediately prior to trauma predicts subsequent psychiatric disorder. Sleep, 33(1), 69-74.

Caldwell, A. J., Knapik, J. J., \& Lieberman, H. R. (2017). Trends and factors associated with insomnia and sleep apnea in all United States military service members from 2005 to 2014. J Sleep Res, 26(5), 665-670. doi:10.1111/jsr.12543

Capaldi, V. F., 2nd, Guerrero, M. L., \& Killgore, W. D. (2011). Sleep disruptions among returning combat veterans from Iraq and Afghanistan. Mil Med, 176(8), 879-888.

Castriotta, R. J., \& Lai, J. M. (2001). Sleep disorders associated with traumatic brain injury. Arch Phys Med Rehabil, 82(10), 1403-1406. doi:10.1053/apmr.2001.26081

Collen, J., Orr, N., Lettieri, C. J., Carter, K., \& Holley, A. B. (2012). Sleep disturbances among soldiers with combatrelated traumatic brain injury. CHEST, 142(3), 622-630. doi:10.1378/chest.11-1603

Congressional Budget Office. (2012). The Veterans Health Administration's Treatment of PTSD and Traumatic Brain Injury Among Recent Combat Veterans. Retrieved from https://www.cbo.gov/publication/42969

de Dassel, T., Wittmann, L., Protic, S., Hollmer, H., \& Gorzka, R. J. (2017). Association of Posttraumatic Nightmares and Psychopathology in a Military Sample. Psychol Trauma. doi:10.1037/tra0000319

Defense and Veterans Brain Injury Center. (2017). DoD worldwide numbers for tbi. Retrieved from httpdvbic.dcoe.mil/dodworldwide-numbers-tbi

Elliott, T. R., Hsiao, Y. Y., Kimbrel, N. A., Meyer, E., DeBeer, B. B., Gulliver, S. B.,... \& Morissette, S. B. (2017). Resilience and Traumatic Brain Injury Among Iraq/Afghanistan War Veterans: Differential Patterns of Adjustment and Quality of Life. J Clin Psychol, 73(9), 1160-1178. doi:10.1002/jclp.22414

Ettenhofer, M. L., Melrose, R. J., Delawalla, Z., Castellon, S. A., \& Okonek, A. (2012). Correlates of functional status among OEF/OIF veterans with a history of traumatic brain injury. Mil Med, 177(11), 1272-1278.

Farrell-Carnahan, L., Franke, L., Graham, C., \& McNamee, S. (2013). Subjective sleep disturbance in veterans receiving care in the Veterans Affairs Polytrauma System following blastrelated mild traumatic brain injury. Mil Med, 178(9), 951-956. doi:10.7205/milmed-d-13-00037
Gehrman, P., Seelig, A. D., Jacobson, I. G., Boyko, E. J., Hooper, T. I., Gackstetter, G. D.,... \& Smith, T. C. (2013). Predeployment Sleep Duration and Insomnia Symptoms as Risk Factors for New-Onset Mental Health Disorders Following Military Deployment. Sleep, 36(7), 1009-1018. doi:10.5665/sleep.2798

Germain, A., Buysse, D. J., \& Nofzinger, E. (2008). Sleep-specific mechanisms underlying posttraumatic stress disorder: Integrative review and neurobiological hypotheses. Sleep Med Rev, 12(3), 185-195. doi:10.1016/j.smrv.2007.09.003

Germain, A., Richardson, R., Stocker, R., Mammen, O., Hall, M., Bramoweth, A. D.,... \& Buysse, D. J. (2014). Treatment for insomnia in combat-exposed OEF/OIF/OND military veterans: Preliminary randomized controlled trial. Behav Res Ther, 61, 78-88. doi:10.1016/j.brat.2014.07.016

Germain, A., Shear, M. K., Hall, M., \& Buysse, D. J. (2007). Effects of a brief behavioral treatment for PTSD-related sleep disturbances: A pilot study. Behav Res Ther, 45(3), 627-632. doi:10.1016/j.brat.2006.04.009

Gilbert, K. S., Kark, S. M., Gehrman, P., \& Bogdanova, Y. (2015). Sleep disturbances, TBI and PTSD: Implications for treatment and recovery. Clin Psychol Rev, 40, 195-212. doi:10.1016/j.cpr.2015.05.008

Gill, J., Lee, H., Barr, T., Baxter, T., Heinzelmann, M., Rak, H., \& Mysliwiec, V. (2014). Lower health related quality of life in U.S. military personnel is associated with service-related disorders and inflammation. Psychiatry Res, 216(1), 116-122. doi:10.1016/j.psychres.2014.01.046

Holster, J. L., Bryan, C. J., Heron, E. A., \& Seegmiller, R. A. (2017). Traumatic Brain Injury, Sleep, and Mental Health: A Longitudinal Study of Air Force Personnel Pre- and Postdeployment to Iraq. J Head Trauma Rehabil, 32(1), 25-33. doi:10.1097/htr.0000000000000237

James, L. M., Strom, T. Q., \& Leskela, J. (2014). Risk-taking behaviors and impulsivity among veterans with and without PTSD and mild TBI. Military medicine, 179(4), 357-363.

Jaramillo, C. A., Cooper, D. B., Wang, C. P., Tate, D. F., Eapen, B. C., York, G. E., \& Pugh, M. J. (2015). Subgroups of US IRAQ and Afghanistan veterans: Associations with traumatic brain injury and mental health conditions. Brain Imaging Behav, 9(3), 445-455. doi:10.1007/s11682-015-9402-8

Kempf, J., Werth, E., Kaiser, P. R., Bassetti, C. L., \& Baumann, C. R. (2010). Sleep-wake disturbances 3 years after traumatic brain injury. J Neurol Neurosurg Psychiatry, 81(12), 14021405. doi:10.1136/jnnp.2009.201913

Lang, K. P., Veazey-Morris, K., \& Andrasik, F. (2014). Exploring the role of insomnia in the relation between PTSD and pain in veterans with polytrauma injuries. J Head Trauma Rehabil, 29(1), 44-53. doi:10.1097/HTR.0b013e31829c85d0

Lew, H. L., Pogoda, T. K., Hsu, P. T., Cohen, S., Amick, M. M., Baker, E.,... \& Vanderploeg, R. D. (2010). Impact of the "polytrauma clinical triad" on sleep disturbance in a department of veterans affairs outpatient rehabilitation setting. Am J Phys Med Rehabil, 89(6), 437-445. doi:10.1097/ PHM.0b013e3181ddd301

Lippa, S. M., Fonda, J. R., Fortier, C. B., Amick, M. A., Kenna, A., Milberg, W. P., \& McGlinchey, R. E. (2015). Deployment-related psychiatric and behavioral conditions and their association with functional disability in OEF/OIF/OND veterans. J Trauma Stress, 28(1), 25-33. doi:10.1002/jts. 21979

Macera, C. A., Aralis, H. J., Rauh, M. J., \& MacGregor, A. J. (2013). Do sleep problems mediate the relationship 
between traumatic brain injury and development of mental health symptoms after deployment? Sleep, 36(1), 83-90. doi: $10.5665 /$ sleep. 2306

Maguen, S., Lau, K. M., Madden, E., \& Seal, K. (2012). Relationship of screen-based symptoms for mild traumatic brain injury and mental health problems in Iraq and Afghanistan veterans: Distinct or overlapping symptoms? J Rehabil Res Dev, 49(7), $1115-1126$

Mathias, J. L., \& Alvaro, P. K. (2012). Prevalence of sleep disturbances, disorders, and problems following traumatic brain injury: A meta-analysis. Sleep Med, 13(7), 898-905. doi:10.1016/j.sleep.2012.04.006

Matthews, E. E., Signoracci, G. M., Stearns-Yoder, K., \& Brenner, L. A. (2016). A Qualitative Study of Sleep-Wake Disturbance Among Veterans With Post-Acute Moderate to Severe Traumatic Brain Injury. J Head Trauma Rehabil, 31(2), 126-135. doi:10.1097/htr.0000000000000216

Mysliwiec, V., Gill, J., Lee, H., Baxter, T., Pierce, R., Barr, T. L.,... \& Roth, B. J. (2013). Sleep disorders in US military personnel: A high rate of comorbid insomnia and obstructive sleep apnea. CHEST, 144(2), 549-557. doi:10.1378/chest.130088

Mysliwiec, V., McGraw, L., Pierce, R., Smith, P., Trapp, B., \& Roth, B. J. (2013). Sleep disorders and associated medical comorbidities in active duty military personnel. Sleep, 36(2), 167-174. doi:10.5665/sleep. 2364

Norris, J. N., Sams, R., Lundblad, P., Frantz, E., \& Harris, E. (2014). Blast-related mild traumatic brain injury in the acute phase: Acute stress reactions partially mediate the relationship between loss of consciousness and symptoms. Brain Inj, 28(8), 1052-1062. doi:10.3109/02699052.2014.891761

Orff, H. J., Ayalon, L., \& Drummond, S. P. (2009). Traumatic brain injury and sleep disturbance: A review of current research. J Head Trauma Rehabil, 24(3), 155-165. doi:10.1097/HTR.0b013e3181a0b281

Peters, M. D., Godfrey, C. M., Khalil, H., McInerney, P., Parker, D., \& Soares, C. B. (2015). Guidance for conducting systematic scoping reviews. Int J Evid Based Healthc, 13(3), 141-146. doi:10.1097/xeb.0000000000000050

Peterson, A. L., Goodie, J. L., Satterfield, W. A., \& Brim, W. L. (2008). Sleep disturbance during military deployment. Mil Med, 173(3), 230-235.

Petrie, E. C., Cross, D. J., Yarnykh, V. L., Richards, T., Martin, N. M., Pagulayan, K.,... \& Peskind, E. R. (2014). Neuroimaging, behavioral, and psychological sequelae of repetitive combined blast/impact mild traumatic brain injury in Iraq and Afghanistan war veterans. J Neurotrauma, 31(5), 425-436. doi:10.1089/neu.2013.2952

Ponsford, J. L., Ziino, C., Parcell, D. L., Shekleton, J. A., Roper, M., Redman, J. R.,... \& Rajaratnam, S. M. (2012). Fatigue and sleep disturbance following traumatic brain injury-their nature, causes, and potential treatments. J Head Trauma Rehabil, 27(3), 224-233. doi:10.1097/HTR.0b013e31824ee1a8

Pugh, M. J., Finley, E. P., Copeland, L. A., Wang, C. P., Noel, P. H., Amuan, M. E.,... \& Pugh, J. A. (2014). Complex comorbidity clusters in OEF/OIF veterans: The polytrauma clinical triad and beyond. Med Care, 52(2), 172-181. doi: $10.1097 / \mathrm{mlr} .0000000000000059$

Robinson, M. E., Lindemer, E. R., Fonda, J. R., Milberg, W. P., McGlinchey, R. E., \& Salat, D. H. (2015). Close-range blast exposure is associated with altered functional con- nectivity in Veterans independent of concussion symptoms at time of exposure. Hum Brain Mapp, 36(3), 911-922. doi: $10.1002 / \mathrm{hbm} .22675$

Ruff, R. L., Riechers, R. G., 2nd, Wang, X. F., Piero, T., \& Ruff, S. S. (2012). For veterans with mild traumatic brain injury, improved posttraumatic stress disorder severity and sleep correlated with symptomatic improvement. J Rehabil Res Dev, 49(9), 1305-1320.

Ruff, R. L., Ruff, S. S., \& Wang, X. F. (2009). Improving sleep: Initial headache treatment in OIF/OEF veterans with blastinduced mild traumatic brain injury. J Rehabil Res Dev, 46(9), 1071-1084.

Sandsmark, D. K., Elliott, J. E., \& Lim, M. M. (2017). Sleep-wake disturbances after traumatic brain injury: Synthesis of human and animal studies. Sleep, 40(5).

Schiehser, D. M., Delano-Wood, L., Jak, A. J., Hanson, K. L., Sorg, S. F., Orff, H., \& Clark, A. L. (2017). Predictors of cognitive and physical fatigue in post-acute mild-moderate traumatic brain injury. Neuropsychol Rehabil, 27(7), 10311046. doi:10.1080/09602011.2016.1215999

Shekleton, J. A., Parcell, D. L., Redman, J. R., PhippsNelson, J., Ponsford, J. L., \& Rajaratnam, S. M. (2010). Sleep disturbance and melatonin levels following traumatic brain injury. Neurology, 74(21), 1732-1738. doi:10.1212/WNL.0b013e3181e0438b

Si, B., Dumkrieger, G., Wu, T., Zafonte, R., Valadka, A. B., Okonkwo, D. O.,... \& Li, J. (2018). Sub-classifying patients with mild traumatic brain injury: A clustering approach based on baseline clinical characteristics and 90-day and 180-day outcomes. PloS one, 13(7), e0198741.

Stocker, R. P., Cieply, M. A., Paul, B., Khan, H., Henry, L., Kontos, A. P., \& Germain, A. (2014). Combat-related blast exposure and traumatic brain injury influence brain glucose metabolism during REM sleep in military veterans. Neurolmage, 99, 207214. doi:10.1016/j.neuroimage.2014.05.067

Stocker, R. P., Paul, B. T., Mammen, O., Khan, H., Cieply, M. A., \& Germain, A. (2016). Effects of Blast Exposure on Subjective and Objective Sleep Measures in Combat Veterans with and without PTSD. J Clin Sleep Med, 12(1), 49-56. doi: $10.5664 /$ jesm.5392

Tanev, K. S., Pentel, K. Z., Kredlow, M. A., \& Charney, M. E. (2014). PTSD and TBI co-morbidity: Scope, clinical presentation and treatment options. Brain Inj, 28(3), 261-270. doi:10.3109/02699052.2013.873821

Thurman, D. J., Alverson, C., Dunn, K. A., Guerrero, J., \& Sniezek, J. E. (1999). Traumatic brain injury in the United States: A public health perspective. J Head Trauma Rehabil, 14(6), 602615

Troxel, W. M., Shih, R. A., Pedersen, E. R., Geyer, L., Fisher, M. P., Griffin, B. A.,... \& Steinberg, P. S. (2015). Sleep in the military: Promoting healthy sleep among US servicemembers. Rand health quarterly, 5(2).

Verfaellie, M., Lee, L. O., Lafleche, G., \& Spiro, A. (2016). Self-Reported Sleep Disturbance Mediates the Relationship Between PTSD and Cognitive Outcome in Blast-Exposed OEF/OIF Veterans. J Head Trauma Rehabil, 31(5), 309-319. doi:10.1097/htr.0000000000000197

Vuletic, S., Bell, K. R., Jain, S., Bush, N., Temkin, N., Fann, J. R.,... \& Gahm, G. A. (2016). Telephone Problem-Solving Treatment Improves Sleep Quality in Service Members With Combat-Related Mild Traumatic Brain Injury: Results From 
a Randomized Clinical Trial. J Head Trauma Rehabil, 31(2), 147-157. doi:10.1097/htr.0000000000000221

Waldron-Perrine, B., Hennrick, H., Spencer, R. J., Pangilinan, P. H., \& Bieliauskas, L. A. (2014). Postconcussive symptom report in polytrauma: Influence of mild traumatic brain injury and psychiatric distress. Mil Med, 179(8), 856-864. doi:10.7205/milmed-d-13-00282

Wallace, D. M., Shafazand, S., Ramos, A. R., Carvalho, D. Z., Gardener, H., Lorenzo, D., \& Wohlgemuth, W. K. (2011). Insomnia characteristics and clinical correlates in Operation
Enduring Freedom/Operation Iraqi Freedom veterans with post-traumatic stress disorder and mild traumatic brain injury: An exploratory study. Sleep Med, 12(9), 850-859. doi:10.1016/j.sleep.2011.06.004

Wiseman-Hakes, C., Murray, B., Moineddin, R., Rochon, E., Cullen, N., Gargaro, J., \& Colantonio, A. (2013). Evaluating the impact of treatment for sleep/wake disorders on recovery of cognition and communication in adults with chronic TBI. Brain injury, 27(12), 1364-1376. 